\title{
Theory of charge transport in diffusive normal metal/unconventional singlet superconductor contacts
}

\author{
Y. Tanaka, ${ }^{1,2,3}$ Yu. V. Nazarov, ${ }^{3}$ A. A. Golubov, ${ }^{4}$ and S. Kashiwaya ${ }^{5}$ \\ ${ }^{1}$ Department of Applied Physics, Nagoya University, Nagoya 464-8603, Japan \\ ${ }^{2}$ CREST Japan Science and Technology Cooperation (JST), Nagoya 464-8603, Japan \\ ${ }^{3}$ Department of Nanoscience, Faculty of Applied Sciences, Delft University of Technology, 2628 CJ Delft, The Netherlands \\ ${ }^{4}$ Faculty of Science and Technology, University of Twente, 7500 AE Enschede, The Netherlands \\ ${ }^{5}$ National Institute of Advanced Industrial Science and Technology, Tsukuba 305-8568, Japan
}

(Received 22 November 2003; published 23 April 2004)

\begin{abstract}
We analyze the transport properties of contacts between unconventional superconductor and normal diffusive metal in the framework of the extended circuit theory. We obtain a general boundary condition for the Keldysh-Nambu Green's functions at the interface that is valid for arbitrary transparencies of the interface. This allows us to investigate the voltage-dependent conductance (conductance spectrum) of a diffusive normal metal (DN)/ unconventional singlet superconductor junction in both ballistic and diffusive cases. For $d$-wave superconductors, we calculate conductance spectra numerically for different orientations of the junctions, resistances, Thouless energies in DN, and transparencies of the interface. We demonstrate that conductance spectra exhibit a variety of features including a V-shaped gaplike structure, zero bias conductance peak (ZBCP) and zero bias conductance dip. We show that two distinct mechanisms: (i) coherent Andreev reflection (CAR) in DN and (ii) formation of midgap Andreev bound state at the interface of $d$-wave superconductors, are responsible for ZBCP, their relative importance being dependent on the angle $\alpha$ between the interface normal and the crystal axis of $d$-wave superconductors. For $\alpha=0$, the ZBCP is due to CAR in the junctions of low transparency with small Thouless energies. This is similar to the case of diffusive normal metal/insulator/ $s$-wave superconductor junctions. With increase of $\alpha$ from zero to $\pi / 4$, the MABS contribution to ZBCP becomes more prominent and the effect of CAR is gradually suppressed. Such complex spectral features shall be observable in conductance spectra of realistic high- $T_{c}$ junctions at very low temperature.
\end{abstract}

DOI: 10.1103/PhysRevB.69.144519

PACS number(s): 74.50.+r, 74.20.Rp, 74.70.Kn

\section{INTRODUCTION}

The low-energy transport in mesoscopic superconducting systems is governed by Andreev reflection, ${ }^{1}$ a unique process specific to electron scattering at normal metal/ superconductor interfaces. The phase coherence between incoming electrons and Andreev reflected holes persists at a mesoscopic length scale in the diffusive normal metal, which enhances interference effects on the probability of Andreev reflection. ${ }^{2}$ The coherence plays an important role at sufficiently low temperatures and voltages when the energy broadening due to either voltage or temperature becomes of the order of the Thouless energy $E_{T h}$ of the mesoscopic structure. As a result, the conductance spectra of mesoscopic junctions may be significantly modified by these interference effects. A remarkable experimental manifestation of the electron-hole phase coherence is the observation of the zero bias conductance peak (ZBCP) in diffusive normal metal $(\mathrm{DN}) /$ superconductor $(\mathrm{S})$ tunneling junctions. ${ }^{3-13}$

Various theoretical models of charge transport in diffusive junctions extend the clean limit theories developed by Blonder, Tinkham, and Klapwijk ${ }^{14}$ (BTK) and Zaitsev. ${ }^{15}$ In Refs. 16-21 the scattering matrix approach was used. On the other hand, the quasiclassical Green's function method in nonequilibrium superconductivity ${ }^{22}$ is much more powerful and convenient for the actual calculations of conductance for the arbitrary bias voltages. ${ }^{23}$ Using the Kuprianov and Lukichev boundary condition ${ }^{24}$ for a diffusive SIN interface,
Volkov, Zaitsev, and Klapwijk have obtained the conductance spectra with ZBCP, origin of which was attributed to coherent Andreev reflection (CAR) which induces the proximity effect in diffusive metal. ${ }^{23}$ Several authors studied the charge transport in mesoscopic junctions combining this boundary condition with Usadel ${ }^{25}$ equations that describe superconducting correlations in a diffusive metal. ${ }^{26-33}$

The modified boundary conditions were studied by several authors. ${ }^{35,36}$ Important progress was achieved by one of the authors ${ }^{26,37}$ who developed the so-called "circuit theory" for matrix currents that allows one to formulate boundary conditions for Usadel-like equations in the case of arbitrary transparencies. By using this generalized boundary condition, three of the authors have evaluated the conductance in $\mathrm{DN} / \mathrm{S}$ junctions and demonstrated how various may be the conductance spectra. ${ }^{38}$

Another mechanism of ZBCP plays the role in ballistic unconventional superconductor junctions. The conductance peak in this situation arises from the formation of midgap Andreev bound states (MABS) at the interface. ${ }^{34,39-42}$ The experimental observation of the ZBCP has been reported for various unconventional superconductors of anisotropic pairing symmetry. ${ }^{42-57} \mathrm{~A}$ basic theory of ballistic transport in the presence of MABS has been formulated in Refs. 40 and 42. Stimulated by this theory, extensive studies of MABS in unconventional superconductor junctions have been performed during the last decade: in the case of broken time reversal symmetry state, ${ }^{58-65}$ in triplet superconductor junctions, ${ }^{66-71}$ in quasi-one-dimensional organic superconductors, ${ }^{72-74}$ 
MABS and Doppler effect, ${ }^{75-79}$ MABS in ferromagnet junctions, ${ }^{80-88}$ influence of MABS on Josephson effect ${ }^{89-98}$ and other related problems. ${ }^{99-107}$ However, an interesting question remained: how two mechanisms of ZBCP (due to CAR and due to MABS) work together, this being relevant for diffusive normal metal/unconventional superconductor (DN/US) junctions.

To solve this problem, three of the present authors have recently extended the circuit theory to the systems that contain unconventional singlet superconductor junctions. ${ }^{108} \mathrm{Ap}-$ plication of this theory for DN/ $d$-wave superconductor (DN/ d) junctions has shown that the formation of MABS strongly competes with the proximity effect that is an essential ingredient for CAR in DN. The MABS induces the unconventional channels where quasiparticles are resonantly transmitted through the interface. The overall contribution of these channels to the proximity effect is, however, suppressed by the isotropization, i.e., the angular averaging over momentum directions of injected quasiparticles. However, Ref. 108 does not contain the necessary technical details of the matrix current derivation and presents the results only for low voltage limit. To compare with experiment, one has to evaluate the conductance spectrum in wide range of bias voltage.

In this paper, we present a detailed derivation of the matrix current in DN/US junctions. Although the relation obtained is valid for both singlet and triplet superconductor junctions, we focus on the case of singlet superconductor. We present detailed numerical calculations of the conductance spectra of DN/US junctions for $d$-wave superconductors. We investigate the dependence of the spectra on various parameters: the height of the barrier at the interface, resistance $R_{d}$ in DN, the Thouless energy $E_{T h}$ in DN, and the angle between the normal to the interface and the crystal axis of $d$-wave superconductor $(\alpha)$. We normalize the voltagedependent conductance $\sigma_{S}(\mathrm{eV})$ by its value in the normal state, $\sigma_{N}$, so that $\sigma_{T}(e V)=\sigma_{S}(e V) / \sigma_{N}$.

Our main results are as follows:

(1) The ZBCP is frequently seen in the shape of $\sigma_{T}(e V)$. For $\alpha \neq 0$, the ZBCP is robust, not depending on the diffusive resistance $R_{d}$. For $\alpha=0$, ZBCP is due to the CAR.

(2) The appearance of ZBCP is different for MABS and CAR mechanisms. The first mechanism may lead to arbitrarily large $\sigma_{T}(0)$. The second mechanism cannot provide $\sigma_{T}(0)$ exceeding unity. While for the first mechanism the width of the ZBCP is determined by the transparency of the junction, it is determined by Thouless energy for the second one. These two mechanisms compete since the proximity effect and the MABS in singlet junctions are generally incompatible. ${ }^{108}$

(3) In the extreme case $\alpha=\pi / 4$ the proximity effect and the CAR are absent. The $\sigma_{T}(e V)$ is then given by a simple Ohm's law: $\sigma_{T}(e V)=\left(R_{b}+R_{d}\right) /\left(R_{R_{d}=0}+R_{d}\right) R_{b}$ being the resistance of the interface.

(4) For $\alpha=0$, when MABS are absent for $R_{d}=0$, the $\mathrm{ZBCP}$ of $\sigma_{T}(e V)$ is attributed to the CAR alone. When the transparency of the junction is sufficiently low, $\sigma_{T}(e V)$ for $|e V|<\Delta_{0}$ is enhanced with the increase of $R_{d}$ due to the enhancement of the proximity effect $\left(\Delta_{0}\right.$ is the maximum amplitude of the pair potential). The ZBCP becomes prominent for $E_{T h} \ll \Delta_{0}$ and $R_{d} / R_{b}<1\left(R_{d} \neq 0\right)$. In this case, the ZBCP turns into a zero-bias conductance dip (ZBCD) with a further increase of $R_{d} / R_{b}$.

(5) We have shown that the conductance spectrum can vary substantially depending on the parameters. The results obtained are important to analyze the actual experimental data on conductance spectra of high- $T_{C}$ cuprate junctions since in this case the diffusive scattering in the normal metal is of special relevance.

The structure of the paper is as follows. We formulate the model in use in Sec. II. We also present there the detailed derivation of the matrix current and end up with the expression for the normalized conductance. We focus on $d$-wave superconductor junctions in Sec. III and evaluate $\sigma_{T}(e V)$ and the measure of the proximity effect $\theta_{0}$ for various cases. We summarize the results in Sec. IV.

\section{FORMULATION}

In this section we introduce the model and the formalism. We consider a junction consisting of normal and superconducting reservoirs connected by a quasi-one-dimensional diffusive conductor (DN) with a length $L$ much larger than the mean-free path. The interface between the DN conductor and the US (unconventional superconductor) electrode has a resistance $R_{b}$ while the DN/N interface has zero resistance. The positions of the DN/N interface and the DN/S interface are denoted as $x=-L$ and $x=0$, respectively. According to the circuit theory, ${ }^{37}$ the constriction area $\left(-L_{1}<x<L_{1}\right)$ between DN and US is considered as composed of the diffusive isotropization zone $\left(-L_{1}<x<-L_{2}\right)$, the left side ballistic zone $\left(-L_{2}<x<0\right)$, the right side ballistic zone $(0<x$ $\left.<L_{1}\right)$, and the scattering zone $(x=0)$. The scattering zone is modeled as an insulating $\delta$-function barrier with the transparency $T_{n}=4 \cos ^{2} \phi /\left(4 \cos ^{2} \phi+Z^{2}\right)$, where $Z$ is a dimensionless constant, $\phi$ is the injection angle measured from the interface normal to the junction, and $n$ is the channel index. We assume that the sizes of the ballistic and scattering zones along $x$ axis are much shorter than the superconducting coherence length.

Here, we express insulating barrier as a $\delta$-function model $H \delta(x)$, where $Z$ is given by $Z=2 m H /\left(\hbar^{2} k_{F}\right)$ with Fermi momentum $k_{F}$ and effective mass $m$. In order to clarify charge transport in DN/US junctions, we must obtain Keldysh-Nambu Green's function, which has indices of transport channels and the direction of motion along $x$ axis taking into account the proper boundary conditions. For this purpose it is necessary to extend a general theory of boundary condition which covers the crossover from ballistic to diffusive $\operatorname{cases}^{37}$ formulated for conventional junctions in the framework of the circuit theory. ${ }^{26,37}$ However, the circuit theory cannot be directly applied to unconventional superconductors since it requires the isotropization. To avoid this difficulty we restrict the discussion to a conventional model of smooth interface by assuming momentum conservation in the plane of the interface.

In the following sections, we will show how to derive the matrix current in DN/US junctions. Then we will derive the 
retarded and Keldysh components of the matrix current. Finally, we will show how to calculate conductance of the junctions.

\section{A. Calculation of the matrix current}

To derive the relation between the matrix current and Green's functions, we make use of the method proposed in Ref. 37. The method puts the older ideas ${ }^{15}$ to the framework of Landauer-Büttiker scattering formalism. One expresses the matrix current in a constriction in terms of onedimensional Green's functions ${\stackrel{\check{g}}{n, \sigma ; n^{\prime}, \sigma^{\prime}}}\left(\varepsilon ; x, x^{\prime}\right)$, where $n$, $n^{\prime}$ and $\sigma, \sigma^{\prime}= \pm 1$ denote the indices of transport channels and the direction of motion along $x$ axis, respectively. The "check" represents the Keldysh-Nambu structure. These Green's functions have to be expressed in terms of the transfer matrix that incorporates all information about the scattering, and asymptotic Green's functions presenting boundary conditions deep in each side of the constriction. Here, we restrict the discussion to a conventional model of smooth interface, assuming momentum conservation in the plane of the interface. Within the model, the channel number eventually numbers possible values of this in-plane momentum and the transfer matrix becomes block diagonal in the channel index. Following the treatment developed in Ref. 37, we thus solve Green's functions $\check{g}_{n, \sigma ; n^{\prime}, \sigma^{\prime}}\left(\varepsilon, x, x^{\prime}\right)$ separately for each channel. $\check{g}_{n, \sigma ; n^{\prime}, \sigma^{\prime}}\left(\varepsilon, x, x^{\prime}\right)$ can be expressed as

$$
\begin{aligned}
\check{g}_{n, \sigma ; n^{\prime}, \sigma^{\prime}}\left(\varepsilon, x, x^{\prime}\right)= & \sum_{\sigma, \sigma^{\prime}= \pm 1} \exp \left(i \sigma p_{n} x-i \sigma^{\prime} p_{n} x^{\prime}\right) \\
& \times \check{G}_{n}^{\sigma, \sigma^{\prime}}\left(x, x^{\prime}\right) .
\end{aligned}
$$

The functions $\check{G}_{n}^{\sigma, \sigma^{\prime}}\left(x, x^{\prime}\right)$ are varying smoothly at the scale of $1 / p_{n}$ and obey the following semiclassical equation:

$$
\left(i \sigma v_{n} \frac{\partial}{\partial x}+\check{H}(x)\right) \check{G}_{n}^{\sigma, \sigma^{\prime}}\left(x, x^{\prime}\right)=0
$$

with effective Hamiltonian $\check{H}(x)$ where $\check{H}(x)$ and $\check{H}_{0}(x)$ are given by

$$
\begin{gathered}
\check{H}(x)=\check{H}_{0}(x)-\check{\Sigma}_{i m p}(x), \\
\check{H}_{0}(x)=\varepsilon \check{\tau}_{z}+\check{\Delta}(x), \quad \check{\tau}_{z}=\left(\begin{array}{cc}
\hat{\tau}_{z} & 0 \\
0 & \hat{\tau}_{z}
\end{array}\right) .
\end{gathered}
$$

In the above, $\check{\Sigma}_{\text {imp }}(x)$ is a self-energy due to the impurity scattering and $\Sigma_{i m p}(x) \neq 0$ is satisfied only for $x<0$. The self-energy originating from the superconducting pair potential $\check{\Delta}(x)=0$ for $x<0$ while $\check{\Delta}(x)$ for $x>0$ is given by

$$
\check{\Delta}(x)=\left(\begin{array}{cc}
\hat{\Delta}_{\sigma} & 0 \\
0 & \hat{\Delta}_{\sigma}
\end{array}\right), \quad \hat{\Delta}_{\sigma}=\left(\begin{array}{cc}
0 & \Delta_{\sigma} \\
-\Delta_{\sigma}^{*} & 0
\end{array}\right) .
$$

$\check{G}_{n}^{\sigma \sigma^{\prime}}\left(x, x^{\prime}\right)$ has discontinuity at $x=x^{\prime}$,

$$
\check{G}_{n}^{\sigma \sigma^{\prime}}(x+0, x)-\check{G}_{n}^{\sigma \sigma^{\prime}}(x-0, x)=-i \check{\Gamma} \sigma \delta_{\sigma \sigma^{\prime}} /\left|v_{n}\right| .
$$

To get rid of the discontinuity, we define $\bar{g}_{n}^{\sigma \sigma^{\prime}}\left(x, x^{\prime}\right)$ as follows:

$$
2 i \check{G}_{n}^{\sigma \sigma^{\prime}}\left(x, x^{\prime}\right)=\left[\bar{g}_{n}^{\sigma \sigma^{\prime}}\left(x, x^{\prime}\right)+\sigma \operatorname{sgn}\left(x-x^{\prime}\right)\right] /\left|v_{n}\right| .
$$

We denote $\bar{g}_{n}^{\sigma \sigma^{\prime}}\left(0_{+}, 0_{+}\right)=\bar{g}_{2}$ and $\bar{g}_{n}^{\sigma \sigma^{\prime}}\left(0_{-}, 0_{-}\right)=\bar{g}_{1}$, where $\bar{g}_{2}$ and $\bar{g}_{1}$ satisfy

$$
\bar{g}_{2}=\bar{M}^{\dagger} \bar{g}_{1} \bar{M},
$$

using a transfer matrix $\bar{M}$.

The derivation of the matrix current

$$
\check{I}=\frac{2 e^{2}}{h} \operatorname{Tr}_{n, \sigma}\left[\bar{\Sigma}^{z} \bar{g}_{1}\right]=\frac{2 e^{2}}{h} \operatorname{Tr}_{n, \sigma}\left[\bar{\Sigma}^{z} \bar{g}_{2}\right]
$$

is given in the Appendix, where it is shown that $\breve{I}_{n 0}$ can be represented as follows: ${ }^{108}$

$$
\check{I}_{n 0}=2\left[\check{G}_{1}, \check{B}_{n}\right]
$$

with

$$
\begin{aligned}
\check{B}_{n}= & \left(-T_{1 n}\left[\check{G}_{1}, \check{H}_{-}^{-1}\right]+\check{H}_{-}^{-1} \check{H}_{+}-T_{1 n}^{2} \check{G}_{1} \check{H}_{-}^{-1} \check{H}_{+} \check{G}_{1}\right)^{-1} \\
& \times\left[T_{1 n}\left(1-\check{H}_{-}^{-1}\right)+T_{1 n}^{2} \check{G}_{1} \check{H}_{-}^{-1} \check{H}_{+}\right] .
\end{aligned}
$$

This is the very equation that was first derived as Eq. (2) of Ref. 108. It should be remarked that this formula of the matrix current is very general since it is available both for singlet and triplet superconductors. For low transparency limit, i.e., $T_{n} \ll 1, T_{1 n} \ll 1, \breve{I}_{n 0}$ can be approximated to be

$$
\check{I}_{n 0}=\frac{T_{n}}{2}\left[\check{H}_{+}^{-1}\left(1-\check{H}_{-}\right), \check{G}_{1}\right] .
$$

Equation (10) can be regarded as an extended version of Kuprianov and Lukichev's boundary condition ${ }^{24}$ for unconventional superconductor junctions. On the other hand, when $\check{G}_{2+}=\check{G}_{2-}$ is satisfied as in the conventional superconductor, since

$$
\begin{aligned}
\lim _{\check{H}_{-} \rightarrow 0} & -\check{D}^{-1}\left\{T_{1 n}\left(2 \check{G}_{1}-\left[\check{H}_{-}^{-1}, \check{G}_{1}\right]_{+}\right)+\check{H}_{-}^{-1} \check{H}_{+}\right. \\
& \left.+T_{1 n}^{2} \check{G}_{1} \check{H}_{-}^{-1} \check{H}_{+} \check{G}_{1}\right\} \\
& =\left(\check{H}_{+}+T_{1 n} \check{G}_{1}\right)^{-1}\left(-\check{H}_{+}+T_{1 n} \check{G}_{1}\right)
\end{aligned}
$$

and

$$
\begin{aligned}
& \lim _{\check{H}_{-} \rightarrow 0} \check{G}_{1} \check{D}^{-1} \check{G}_{1}\left\{T_{1 n}\left(2 \check{G}_{1}-\left[\check{H}_{-}^{-1}, \check{G}_{1}\right]_{+}\right)+T_{1 n}^{2} \check{H}_{-}^{-1} \check{H}_{+}\right. \\
& \left.\quad+\check{G}_{1} \check{H}_{-}^{-1} \check{H}_{+} \check{G}_{1}\right\}=\left(\check{G}_{1}+T_{1 n} \check{H}_{+}\right)^{-1}\left(-T_{1 n} \check{H}_{+}+\check{G}_{1}\right)
\end{aligned}
$$

are satisfied, then the resulting $\check{I}_{n 0}$ and $\check{I}$ is reduced to be 


$$
\begin{gathered}
\check{I}_{n 0}=2 T_{1 n}\left(1+T_{1 n}^{2}+T_{1 n}\left[\check{H}_{+}, \check{G}_{1}\right]_{+}\right)^{-1}\left[\check{H}_{+}, \check{G}_{1}\right], \\
\check{I}=\sum_{n} \frac{2 e^{2}}{h}\left(1+T_{1 n}^{2}+T_{1 n}\left[\check{H}_{+}, \check{G}_{1}\right]_{+}\right)^{-1}\left[\check{H}_{+}, \check{G}_{1}\right],
\end{gathered}
$$

which is identical to Eq. (36) of Ref. 37. In the above, the definition of $\check{D}$ is given in the Appendix.

\section{B. Calculation of the retarded part of the matrix current}

In order to calculate the retarded part of the matrix current, we denote Keldysh-Nambu Green's function $\check{G}_{1}, \check{G}_{2 \pm}$,

$$
\check{G}_{1}=\left(\begin{array}{cc}
\hat{R}_{1} & \hat{K}_{1} \\
0 & \hat{A}_{1}
\end{array}\right), \quad \check{G}_{2 \pm}=\left(\begin{array}{cc}
\hat{R}_{2 \pm} & \hat{K}_{2 \pm} \\
0 & \hat{A}_{2 \pm}
\end{array}\right),
$$

where the Keldysh component $\hat{K}_{1,2 \pm}$ is given by $\hat{K}_{1(2 \pm)}$ $=\hat{R}_{1(2 \pm)} \hat{f}_{1(2)}(0)-\hat{f}_{1(2)}(0) \hat{A}_{1(2 \pm)}$ with the retarded component $\hat{R}_{1,2 \pm}$ and the advanced component $\hat{A}_{1,2 \pm}$ using distribution function $\hat{f}_{1(2)}(0)$. In the above, $\hat{R}_{2 \pm}$ is expressed by

$$
\hat{R}_{2 \pm}=\left(g_{ \pm} \hat{\tau}_{3}+f_{ \pm} \hat{\tau}_{2}\right)
$$

with $g_{ \pm}=\varepsilon / \sqrt{\varepsilon^{2}-\Delta_{ \pm}^{2}}, \quad f_{ \pm}=\Delta_{ \pm} / \sqrt{\Delta_{ \pm}^{2}-\varepsilon^{2}}, \quad$ and $\hat{A}_{2 \pm}$ $=-\hat{\tau}_{3} \hat{R}_{2 \pm}^{\dagger} \hat{\tau}_{3}$, where $\varepsilon$ denotes the quasiparticle energy measured from the Fermi energy. $\hat{f}_{2}(0)=f_{0 S}(0)$ $=\tanh \left[\varepsilon /\left(2 k_{\mathrm{B}} T\right)\right]$ in thermal equilibrium with temperature $T$. Here, we put the electrical potential zero in the US electrode. We also denote $\check{H}_{+}, \check{H}_{-}, \check{B}_{n}, \check{I}$ as follows:

$$
\begin{gathered}
\check{H}_{+}=\left(\begin{array}{cc}
\hat{R}_{p} & \hat{K}_{p} \\
0 & \hat{A}_{p}
\end{array}\right), \quad \check{H}_{-}=\left(\begin{array}{cc}
\hat{R}_{m} & \hat{K}_{m} \\
0 & \hat{A}_{m}
\end{array}\right), \\
\check{B}_{n}=\left(\begin{array}{cc}
\hat{B}_{R} & \hat{B}_{K} \\
0 & \hat{B}_{A}
\end{array}\right), \quad \check{I}=\left(\begin{array}{cc}
\hat{I}_{R} & \hat{I}_{K} \\
0 & \hat{I}_{A}
\end{array}\right) .
\end{gathered}
$$

Hereafter, in the present paper, we focus on the singlet superconductor junction case without broken time reversal symmetry states (BTRSS). For triplet case or singlet one with BTRSS, the situation becomes much more complex ${ }^{109}$ and we will discuss it in a forthcoming paper in detail. In singlet superconductors, we can choose $\hat{R}_{1}=\cos \theta_{0} \hat{\tau}_{3}$ $+\sin \theta_{0} \hat{\tau}_{2}$ to satisfy the boundary condition at the interface. After some algebra, we can obtain $\hat{B}_{R}$ as follows:

$$
\begin{aligned}
\hat{B}_{R}= & -T_{1 n}\left[1+T_{1 n}^{2}+T_{1 n}\left(\hat{R}_{1} \hat{R}_{p}^{-1}+\hat{R}_{p}^{-1} \hat{R}_{1}\right)\right]^{-1} \\
& \times\left[T_{1 n} \hat{R}_{1}+\hat{R}_{p}^{-1}\right],
\end{aligned}
$$

where we have used the relation

$$
\hat{R}_{1} \hat{R}_{m}^{-1} \hat{R}_{p}+\hat{R}_{m}^{-1} \hat{R}_{p} \hat{R}_{1}=0
$$

The resulting $\hat{I}_{R}$ can be written as

$$
\hat{I}_{R}=\frac{2 e^{2}}{h} \sum_{n} \frac{-2 T_{n}\left[\cos \theta_{0}\left(f_{+}+f_{-}\right)-\sin \theta_{0}\left(g_{+}+g_{-}\right)\right]}{\left(2-T_{n}\right)\left(1+g_{+} g_{-}+f_{+} f_{-}\right)+T_{n}\left[\cos \theta_{0}\left(g_{+}+g_{-}\right)+\sin \theta_{0}\left(f_{+}+f_{-}\right)\right]} \hat{\tau}_{3} \hat{\tau}_{2} .
$$

This is one of the central results of this paper.

\section{Calculation of the Keldysh part of the matrix current}

Next, we focus on the Keldysh component. We define

$$
I_{b}=\frac{e^{2}}{h} \sum_{n} \operatorname{Tr}\left[\hat{I}_{K} \hat{\tau}_{3}\right]
$$

After straightforward calculations, $I_{b}$ is given by

$$
\begin{aligned}
I_{b}= & \frac{e^{2}}{h} \sum_{n} \operatorname{Tr}\left\{\hat{\tau}_{3}\left(\hat{R}_{1} \hat{B}_{K}+\hat{R}_{1}^{\dagger} \hat{B}_{K}\right)\right. \\
& -\left[\hat{\tau}_{3}\left(\hat{R}_{1}^{\dagger} \hat{B}_{R}^{\dagger}+\hat{B}_{R} \hat{R}_{1}+\hat{B}_{R}^{\dagger} \hat{R}_{1}+\hat{R}_{1}^{\dagger} \hat{B}_{R}\right)\right] f_{0 N}(0) \\
& \left.-\left[\left(\hat{R}_{1}+\hat{R}_{1}^{\dagger}\right)\left(\hat{B}_{R}+\hat{B}_{R}^{\dagger}\right)\right] f_{3 N}(0)\right\}
\end{aligned}
$$

with $\quad \hat{K}_{1}=\hat{R}_{1} \hat{f}_{1}(0)-\hat{f}_{1}(0) \hat{A}_{1} \quad \hat{f}_{1}(0)=f_{0 N}(0)+f_{3 N}(0) \hat{\tau}_{3}$. Since $\hat{B}_{R}$ and $\hat{R}_{1}$ are proportional to the linear combination of $\hat{\tau}_{2}$ and $\hat{\tau}_{3}$, the second term which is proportional to $f_{0 N}(0)$ disappears. It is necessary to obtain $\hat{B}_{K}$ which is given by

$$
\hat{B}_{K}=\hat{D}_{R}^{-1} \hat{N}_{K}-\hat{D}_{R}^{-1} \hat{D}_{K} \hat{D}_{A}^{-1} \hat{N}_{A}
$$

with

$$
\begin{gathered}
\check{D}=-T_{1 n}\left[\check{G}_{1}, \check{H}_{-}^{-1}\right]+\check{H}_{-}^{-1} \check{H}_{+}-T_{1 n}^{2} \check{G}_{1} \check{H}_{-}^{-1} \check{H}_{+} \check{G}_{1}, \\
\check{D}=\left(\begin{array}{cc}
\hat{D}_{R} & \hat{D}_{K} \\
0 & \hat{D}_{A}
\end{array}\right),
\end{gathered}
$$

where $\hat{N}_{K}$ and $\hat{N}_{A}$ are the Keldysh and advanced parts of $\check{N}$ given by

$$
\check{N}=-T_{1 n} \check{H}_{-}^{-1}+T_{1 n}^{2} \check{G}_{1} \check{H}_{-}^{-1} \check{H}_{+} \quad \check{N}=\left(\begin{array}{cc}
\hat{N}_{R} & \hat{N}_{K} \\
0 & \hat{N}_{A}
\end{array}\right) .
$$

We can express $\hat{N}_{K}$ and $\hat{D}_{K}$ as linear combination of distribution functions $f_{0 S}(0), f_{0 N}(0)$, and $f_{3 N}(0)$ as follows:

$$
\begin{aligned}
& \hat{N}_{K}=\hat{C}_{1} f_{0 S}(0)+\hat{C}_{2} f_{0 N}(0)+\hat{C}_{3} f_{3 N}(0), \\
& \hat{D}_{K}=\hat{C}_{4} f_{0 S}(0)+\hat{C}_{5} f_{0 N}(0)+\hat{C}_{6} f_{3 N}(0),
\end{aligned}
$$


by $2 \times 2$ matrix $C_{i}(i=1, \ldots, 6)$. Taking account of the fact that $\hat{D}_{R}^{-1} \hat{C}_{1}, \hat{D}_{R}^{-1} \hat{C}_{2}, \hat{D}_{R}^{-1} \hat{C}_{4} \hat{D}_{A}^{-1} \hat{N}_{A}$, and $\hat{D}_{R}^{-1} \hat{C}_{5} \hat{D}_{A}^{-1} \hat{N}_{A}$ can be expressed by the linear combination of $\hat{\tau}_{2}$ and $\hat{\tau}_{3}$, while $\hat{D}_{R}^{-1} \hat{C}_{3}$ and $\hat{D}_{R}^{-1} \hat{C}_{6} \hat{D}_{A}^{-1} \hat{N}_{A}$ are proportional to the linear combination of $\hat{1}$ and $\hat{\tau}_{1}$, we can express $I_{b}$ as follows:

$$
\begin{gathered}
I_{b}=\frac{e^{2}}{h} \sum_{n} \operatorname{Tr}\left\{\left(\hat{R}_{1}+\hat{R}_{1}^{\dagger}\right)\left[\hat{B}_{K E} \hat{\tau}_{3}-\left(\hat{B}_{R}+\hat{B}_{R}^{\dagger}\right)\right] f_{3 N}(0)\right\}, \\
\hat{B}_{K E}=\hat{D}_{R}^{-1}\left[\hat{C}_{3}-\hat{C}_{6} \hat{D}_{A}^{-1} \hat{N}_{A}\right]
\end{gathered}
$$

with

$$
\begin{gathered}
\hat{C}_{3}=T_{1 n}^{2}\left(\hat{R}_{1} \hat{\tau}_{3}-\hat{\tau}_{3} \hat{A}_{1}\right) \hat{A}_{m}^{-1} \hat{A}_{p}, \\
\hat{C}_{6}=T_{1 n}\left[-\left(\hat{R}_{1} \hat{\tau}_{3}-\hat{\tau}_{3} \hat{A}_{1}\right) \hat{A}_{m}^{-1}+\hat{R}_{m}^{-1}\left(\hat{R}_{1} \hat{\tau}_{3}-\hat{\tau}_{3} \hat{A}_{1}\right)\right. \\
-T_{1 n}\left(\hat{R}_{1} \hat{\tau}_{3}-\hat{\tau}_{3} \hat{A}_{1}\right) \hat{A}_{m}^{-1} \hat{A}_{p} \hat{A}_{1}-T_{1 n} \hat{R}_{1} \hat{R}_{m}^{-1} \hat{R}_{p} \\
\left.\times\left(\hat{R}_{1} \hat{\tau}_{3}-\hat{\tau}_{3} \hat{A}_{1}\right)\right] .
\end{gathered}
$$

Since the following equations are satisfied:

$$
\begin{gathered}
\hat{D}_{A}^{-1} \hat{N}_{A}=-\tau_{3} \hat{B}_{R}^{\dagger} \tau_{3}, \quad \hat{A}_{m(p)}=-\hat{\tau}_{3} \hat{R}_{m(p)}^{\dagger} \hat{\tau}_{3}, \\
\hat{D}_{R}^{-1}\left(-T_{1 n} \hat{R}_{m}^{-1}+T_{1 n}^{2} \hat{R}_{1} \hat{R}_{m}^{-1} \hat{R}_{p}\right)=\hat{B}_{R},
\end{gathered}
$$

$I_{b}$ is given by

$$
\begin{aligned}
I_{b}= & \frac{e^{2}}{h} \sum_{n}\left[-\left(\hat{R}_{1}+\hat{R}_{1}^{\dagger}\right) \hat{B}_{R}\left(\hat{R}_{1}+\hat{R}_{1}^{\dagger}\right) \hat{B}_{R}^{\dagger}\right. \\
& -\left(\hat{R}_{1}+\hat{R}_{1}^{\dagger}\right)\left(\hat{B}_{R}+\hat{B}_{R}^{\dagger}\right) \\
& +T_{1 n}^{2}\left(\hat{R}_{1}+\hat{R}_{1}^{\dagger}\right) \hat{D}_{R}^{-1}\left(\hat{R}_{1}+\hat{R}_{1}^{\dagger}\right)\left(\hat{R}_{m}^{\dagger}\right)^{-1} \hat{R}_{p}^{\dagger} \\
& +T_{1 n}\left(\hat{R}_{1}+\hat{R}_{1}^{\dagger}\right) \hat{D}_{R}^{-1}\left(\hat{R}_{1}+\hat{R}_{1}^{\dagger}\right)\left(\hat{R}_{m}^{\dagger}\right)^{-1} \hat{B}_{R}^{\dagger} \\
& \left.+T_{1 n}^{2}\left(\hat{R}_{1}+\hat{R}_{1}^{\dagger}\right) \hat{D}_{R}^{-1}\left(\hat{R}_{1}+\hat{R}_{1}^{\dagger}\right)\left(\hat{R}_{m}^{\dagger}\right)^{-1} \hat{R}_{p}^{\dagger} \hat{R}_{1}^{\dagger} \hat{B}_{R}^{\dagger}\right] f_{3 N}(0) .
\end{aligned}
$$

After a simple manipulation, we can show

$$
\begin{gathered}
\hat{B}_{R}\left(\hat{R}_{m}^{-1}+T_{1 n} \hat{R}_{1} \hat{R}_{p} \hat{R}_{m}^{-1}\right)-T_{1 n} \hat{R}_{m}^{-1} \hat{R}_{p} \\
=-T_{1 n} \hat{D}_{R}^{-1}=d_{R}^{-1} T_{1 n} \hat{R}_{m} \hat{R}_{p}^{-1}
\end{gathered}
$$

with

$$
d_{R}=\frac{\left(1+T_{1 n}^{2}\right)\left(1+g_{+} g_{-}+f_{+} f_{-}\right)+2 T_{1 n}\left[\cos \theta_{0}\left(g_{+}+g_{-}\right)+\sin \theta_{0}\left(f_{+}+f_{-}\right)\right]}{1+g_{+} g_{-}+f_{+} f_{-}} .
$$

Then the resulting $I_{b}$ is given by

$$
I_{b}=\frac{e^{2}}{h} \sum_{n}\left[-\left(\hat{R}_{1}+\hat{R}_{1}^{\dagger}\right) \hat{B}_{R}\left(\hat{R}_{1}+\hat{R}_{1}^{\dagger}\right) \hat{B}_{R}^{\dagger}-\left(\hat{R}_{1}+\hat{R}_{1}^{\dagger}\right)\left(\hat{B}_{R}+\hat{B}_{R}^{\dagger}\right)-T_{1 n}^{2}\left(\hat{R}_{1}+\hat{R}_{1}^{\dagger}\right) \hat{D}_{R}^{-1}\left(\hat{R}_{1}+\hat{R}_{1}^{\dagger}\right)\left(\hat{D}_{R}^{\dagger}\right)^{-1}\right] f_{3 N}(0) .
$$

Since $\hat{B}_{R}$ is given as $\hat{B}_{R}=b_{2} \hat{\tau}_{2}+b_{3} \hat{\tau}_{3}$ with

$$
\begin{aligned}
& b_{2}=\frac{-T_{1 n}\left[T_{1 n} \sin \theta_{0}\left(1+g_{+} g_{-}+f_{+} f_{-}\right)+f_{+}+f_{-}\right]}{\left(1+T_{1 n}^{2}\right)\left(1+g_{+} g_{-}+f_{+} f_{-}\right)+2 T_{1 n}\left[\cos \theta_{0}\left(g_{+}+g_{-}\right)+\sin \theta_{0}\left(f_{+}+f_{-}\right)\right]}, \\
& b_{3}=\frac{-T_{1 n}\left[T_{1 n} \cos \theta_{0}\left(1+g_{+} g_{-}+f_{+} f_{-}\right)+g_{+}+g_{-}\right]}{\left(1+T_{1 n}^{2}\right)\left(1+g_{+} g_{-}+f_{+} f_{-}\right)+2 T_{1 n}\left[\cos \theta_{0}\left(g_{+}+g_{-}\right)+\sin \theta_{0}\left(f_{+}+f_{-}\right)\right]},
\end{aligned}
$$

final expression of $I_{b}$ is given by the following equation:

$$
\begin{aligned}
& I_{b}=\frac{2 e^{2}}{h} \sum_{n} \frac{T_{n}}{2} \frac{C_{0} f_{3 N}(0)}{\left|\left(2-T_{n}\right)\left(1+g_{+} g_{-}+f_{+} f_{-}\right)+T_{n}\left[\cos \theta_{0}\left(g_{+}+g_{-}\right)+\sin \theta_{0}\left(f_{+}+f_{-}\right)\right]\right|^{2}}, \\
C_{0}= & T_{n}\left(1+\left|\cos \theta_{0}\right|^{2}+\left|\sin \theta_{0}\right|^{2}\right)\left[\left|g_{+}+g_{-}\right|^{2}+\left|f_{+}+f_{-}\right|^{2}+\left|1+f_{+} f_{-}+g_{+} g_{-}\right|^{2}+\left|f_{+} g_{-} g_{+} f_{-}\right|^{2}\right] \\
& +2\left(2-T_{n}\right) \operatorname{Re}\left\{\left(1+g_{+}^{*} g_{-}^{*}+f_{+}^{*} f_{-}^{*}\right)\left[\left(\cos \theta_{0}+\cos \theta_{0}^{*}\right)\left(g_{+}+g_{-}\right)+\left(\sin \theta_{0}+\sin \theta_{0}^{*}\right)\left(f_{+}+f_{-}\right)\right]\right\} \\
& +4 T_{n} \operatorname{Im}\left(\cos \theta_{0} \sin \theta_{0}^{*}\right) \operatorname{Im}\left[\left(f_{+}+f_{-}\right)\left(g_{+}^{*}+g_{-}^{*}\right)\right] .
\end{aligned}
$$


This is a very general expression which is available for any singlet superconductor without BTRSS. This expression is also one of the central results of this paper. For isotropic limit where $f_{+}=f_{-}$and $g_{+}=g_{-}$is satisfied, we obtain

$$
\begin{aligned}
I_{b}=\frac{2 e^{2}}{h} \sum_{n} \frac{T_{n}^{2} \Lambda_{1}+2 T_{n}\left(2-T_{n}\right) \Lambda_{2}}{2\left|\left(2-T_{n}\right)+T_{n}\left[g_{+} \cos \theta_{0}+f_{+} \sin \theta_{0}\right]\right|^{2}}, \\
\Lambda_{1}=\left(1+\left|\cos \theta_{0}\right|^{2}+\left|\sin \theta_{0}\right|^{2}\right)\left(\left|g_{+}\right|^{2}+\left|f_{+}\right|^{2}+1\right) \\
+4 \operatorname{Im}\left[f_{+} g_{+}^{*}\right] \operatorname{Im}\left[\cos \theta_{0} \sin \theta_{0}^{*}\right],
\end{aligned}
$$

which is identical to Eq. (11) of Ref. 37. On the other hand for the ballistic limit, where $\theta_{0}=0$ is satisfied, we can reproduce the generalized BTK formula, ${ }^{40}$

$$
I_{b}=\frac{2 e^{2}}{h} \sum_{n} \frac{T_{n}\left[1+T_{n}\left|\Gamma_{+}\right|^{2}+\left(T_{n}-1\right)\left|\Gamma_{+} \Gamma_{-}\right|^{2}\right]}{\left|1+\left(T_{n}-1\right) \Gamma_{+} \Gamma_{-}\right|^{2}}
$$

with

$$
\Gamma_{+}=\frac{\Delta_{+}}{\varepsilon+\sqrt{\varepsilon^{2}-\Delta_{+}^{2}}}, \quad \Gamma_{-}=\frac{\Delta_{-}}{\varepsilon+\sqrt{\varepsilon^{2}-\Delta_{-}^{2}}},
$$

where the following equations are satisfied:

$$
\begin{gathered}
\frac{g_{+}+g_{-}}{1+g_{+} g_{-}+f_{+} f_{-}}=\frac{1+\Gamma_{+} \Gamma_{-}}{1-\Gamma_{+} \Gamma_{-}}, \\
\frac{f_{+}+f_{-}}{1+g_{+} g_{-}+f_{+} f_{-}}=\frac{i\left(\Gamma_{+}+\Gamma_{-}\right)}{1-\Gamma_{+} \Gamma_{-}} .
\end{gathered}
$$

\section{Calculation of the conductance}

In the following, we apply the quasiclassical Keldysh formalism for calculation of the conductance. The spatial dependence of $4 \times 4$ Green's function in DN $\check{G}_{N}(x)$ which is expressed in the matrix form as

$$
\check{G}_{N}(x)=\left(\begin{array}{cc}
\hat{R}_{N}(x) & \hat{K}_{N}(x) \\
0 & \hat{A}_{N}(x)
\end{array}\right)
$$

should be determined. The Keldysh component $\hat{K}_{N}(x)$ is given by $\hat{K}_{N}(x)=\hat{R}_{N}(x) \hat{f}_{1}(x)-\hat{f}_{1}(x) \hat{A}_{N}(x)$ with retarded component $\hat{R}_{N}(x)$, advanced component $\hat{A}_{N}(x)$ using distribution function $\hat{f}_{1}(x)$. We put the electrical potential zero in the $S$ electrode. In this case the spatial dependence of $\breve{G}_{N}(x)$ in $\mathrm{DN}$ is determined by the static Usadel equation, ${ }^{25}$

$$
D \frac{\partial}{\partial x}\left[\check{G}_{N}(x) \frac{\partial \check{G}_{N}(x)}{\partial x}\right]+i\left[\check{H}, \check{G}_{N}(x)\right]=0
$$

with the diffusion constant $D$ in DN, where $\check{H}$ is given by

$$
\check{H}=\left(\begin{array}{cc}
\hat{H}_{0} & 0 \\
0 & \hat{H}_{0}
\end{array}\right)
$$

with $\hat{H}_{0}=\varepsilon \hat{\tau}_{3}$.
The boundary condition for $\breve{G}_{N}(x)$ at the DN/S interface is given by

$$
\left.\frac{L}{R_{d}}\left[\check{G}_{N}(x) \frac{\partial \check{G}_{N}(x)}{\partial x}\right]\right|_{x=0_{-}}=-\frac{h}{2 e^{2} R_{b}}\langle\check{I}\rangle .
$$

The average over the various angles of injected particles at the interface is defined as

$$
\langle\check{I}(\phi)\rangle=\int_{-\pi / 2}^{\pi / 2} d \phi \cos \phi \check{I}(\phi) / \int_{-\pi / 2}^{\pi / 2} d \phi T(\phi) \cos \phi
$$

with $\check{I}(\phi)=\check{I}$ and $T(\phi)=T_{n}$. The resistance of the interface $R_{b}$ is given by

$$
R_{b}=\frac{h}{2 e^{2}} \frac{2}{\int_{-\pi / 2}^{\pi / 2} d \phi T(\phi) \cos \phi} .
$$

$\breve{G}_{N}(-L)$ coincides with that in the normal state. The electric current is expressed using $\check{G}_{N}(x)$ as

$$
I_{e l}=\frac{-L}{4 e R_{d}} \int_{0}^{\infty} d \varepsilon \operatorname{Tr}\left[\tau_{3}\left(\check{G}_{N}(x) \frac{\partial \check{G}_{N}(x)}{\partial x}\right)^{K}\right],
$$

where $\left\{\check{G}_{N}(x)\left[\partial \check{G}_{N}(x) / \partial x\right]\right\}^{K}$ denotes the Keldysh component of $\left\{\check{G}_{N}(x)\left[\partial \check{G}_{N}(x) / \partial x\right]\right\}$. In the actual calculation, we introduce a parameter $\theta(x)$ which is a measure of the proximity effect in DN where we denoted $\theta(0)=\theta_{0}$ in the previous subsections. Using $\theta(x), \hat{R}_{N}(x)$ can be denoted as

$$
\hat{R}_{N}(x)=\hat{\tau}_{3} \cos \theta(x)+\hat{\tau}_{2} \sin \theta(x) .
$$

$\hat{A}_{N}(x)$ and $\hat{K}_{N}(x)$ satisfy the following equations: $\hat{A}_{N}(x)$ $=-\tau_{3} \hat{R}_{N}^{\dagger}(x) \hat{\tau}_{3}$, and $\hat{K}_{N}(x)=\hat{R}_{N}(x) \hat{f}_{1}(x)-\hat{f}_{1}(x) \hat{A}_{N}(x)$ with the distribution function $\hat{f}_{1}(x)$ which is given by $\hat{f}_{1}(x)$ $=f_{0 N}(x)+\hat{\tau}_{3} f_{3 N}(x)$. In the above, $f_{3 N}(x)$ is the relevant distribution function which determines the conductance of the junction we are now concentrating on. From the retarded or advanced component of the Usadel equation, the spatial dependence of $\theta(x)$ is determined by the following equation:

$$
D \frac{\partial^{2}}{\partial x^{2}} \theta(x)+2 i \varepsilon \sin [\theta(x)]=0,
$$

while for the Keldysh component we obtain

$$
D \frac{\partial}{\partial x}\left[\frac{\partial f_{3 N}(x)}{\partial x} \cosh ^{2} \theta_{\text {imag }}(x)\right]=0
$$

with $\theta_{\text {imag }}(x)=\operatorname{Im}[\theta(x)]$. At $x=-L$, since $\mathrm{DN}$ is attached to the normal electrode, $\theta(-L)=0$ and $f_{3 N}(-L)=f_{t 0}$ is satisfied with

$$
f_{t 0}=\frac{1}{2}\left\{\tanh \left[(\varepsilon+e V) /\left(2 k_{B} T\right)\right]-\tanh \left[(\varepsilon-e V) /\left(2 k_{B} T\right)\right]\right\},
$$


where $V$ is the applied bias voltage. Next, we focus on the boundary condition at the DN/S interface. Taking the retarded part of Eq. (26), we obtain

$$
\left.\frac{L}{R_{d}} \frac{\partial \theta(x)}{\partial x}\right|_{x=0_{-}}=\frac{\langle F\rangle}{R_{b}}
$$

$$
F=\frac{2 T_{n}\left[\cos \theta_{0}\left(f_{+}+f_{-}\right)-\sin \theta_{0}\left(g_{+}+g_{-}\right)\right]}{\left(2-T_{n}\right)\left(1+g_{+} g_{-}+f_{+} f_{-}\right)+T_{n}\left[\cos \theta_{0}\left(g_{+}+g_{-}\right)+\sin \theta_{0}\left(f_{+}+f_{-}\right)\right]} .
$$

On the other hand, from the Keldysh part of Eq. (26), we obtain

$$
\begin{gathered}
\left.\frac{L}{R_{d}}\left(\frac{\partial f_{3 N}}{\partial x}\right) \cosh ^{2} \theta_{0}\right|_{x=0_{-}}=-\frac{\left\langle I_{b 0}\right\rangle f_{3 N}\left(0_{-}\right)}{R_{b}}, \\
I_{b 0}=\frac{T_{n}}{2} \frac{C_{0} f_{3 N}\left(0_{-}\right)}{\left|\left(2-T_{n}\right)\left(1+g_{+} g_{-}+f_{+} f_{-}\right)+T_{n}\left[\cos \theta_{0}\left(g_{+}+g_{-}\right)+\sin \theta_{0}\left(f_{+}+f_{-}\right)\right]\right|^{2}}, \\
C_{0}=T_{n}\left(1+\left|\cos \theta_{0}\right|^{2}+\left|\sin \theta_{0}\right|^{2}\right)\left[\left|g_{+}+g_{-}\right|^{2}+\left|f_{+}+f_{-}\right|^{2}+\left|1+f_{+} f_{-}+g_{+} g_{-}\right|^{2}+\left|f_{+} g_{-}-g_{+} f_{-}\right|^{2}\right] \\
+2\left(2-T_{n}\right) \operatorname{Re}\left\{\left(1+g_{+}^{*} g_{-}^{*}+f_{+}^{*} f_{-}^{*}\right)\left[\left(\cos \theta_{0}+\cos \theta_{0}^{*}\right)\left(g_{+}+g_{-}\right)+\left(\sin \theta_{0}+\sin \theta_{0}^{*}\right)\left(f_{+}+f_{-}\right)\right]\right\} \\
+4 T_{n} \operatorname{Im}\left(\cos \theta_{0} \sin \theta_{0}^{*}\right) \operatorname{Im}\left[\left(f_{+}+f_{-}\right)\left(g_{+}^{*}+g_{-}^{*}\right)\right] .
\end{gathered}
$$

After a simple manipulation, we can obtain

$$
f_{3 N}\left(0_{-}\right)=\frac{R_{b} f_{t 0}}{R_{b}+\frac{R_{d}\left\langle I_{b 0}\right\rangle}{L} \int_{-L}^{0} \frac{d x}{\cosh ^{2} \theta_{\text {imag }}(x)}} .
$$

Since the electric current $I_{e l}$ can be expressed via $\theta_{0}$ in the following form:

$$
I_{e l}=-\left.\frac{L}{e R_{d}} \int_{0}^{\infty}\left(\frac{\partial f_{3 N}}{\partial x}\right)\right|_{x=0_{-}} \cosh ^{2}\left[\operatorname{Im}\left(\theta_{0}\right)\right] d \varepsilon,
$$

we obtain the following final result for the current:

$$
I_{e l}=\frac{1}{e} \int_{0}^{\infty} d \varepsilon \frac{f_{t 0}}{\frac{R_{b}}{\left\langle I_{b 0}\right\rangle}+\frac{R_{d}}{L} \int_{-L}^{0} \frac{d x}{\cosh ^{2} \theta_{\text {imag }}(x)}} .
$$

Then the total resistance $R$ at zero temperature is given by

$$
R=\frac{R_{b}}{\left\langle I_{b 0}\right\rangle}+\frac{R_{d}}{L} \int_{-L}^{0} \frac{d x}{\cosh ^{2} \theta_{\text {imag }}(x)} .
$$

In the following section, we will discuss the normalized conductance $\sigma_{T}(e V)=\sigma_{S}(e V) / \sigma_{N}(e V)$ where $\sigma_{S(N)}(e V)$ is the voltage-dependent conductance in the superconducting (normal) state given by $\sigma_{S}(e V)=1 / R$ and $\sigma_{N}(e V)=\sigma_{N}=1 /\left(R_{d}\right.$ $+R_{b}$ ), respectively.

It should be remarked that in the present circuit theory, $R_{d} / R_{b}$ can be varied independently of $T_{n}$, i.e., of $Z$, since we can change the magnitude of the constriction area independently. In other words, $R_{d} / R_{b}$ is no more proportional to
$T_{a v}(L / l)$, where $T_{a v}$ is the averaged transmissivity and $l$ is the mean-free path in the diffusive region, respectively. Based on this fact, we can choose $R_{d} / R_{b}$ and $Z$ as independent parameters.

\section{RESULTS}

In this subsection, we focus on the line shapes of the conductance where $d$-wave symmetry is chosen as a pairing symmetry of unconventional superconductor. The pair potentials $\Delta_{ \pm}$are given by $\Delta_{ \pm}=\Delta_{0} \cos [2(\phi \mp \alpha)]$ where $\alpha$ denotes the angle between the normal to the interface and the crystal axis of $d$-wave superconductors and $\Delta_{0}$ is the maximum amplitude of the pair potential. In the above, $\phi$ denotes the injection angle of the quasiparticle measured from the $x$ axis. It is known that quasiparticles with injection angle $\phi$ with $\pi / 4-|\alpha|<|\phi|<\pi / 4+|\alpha|$ feel the MABS at the interface which induces ZBCP.

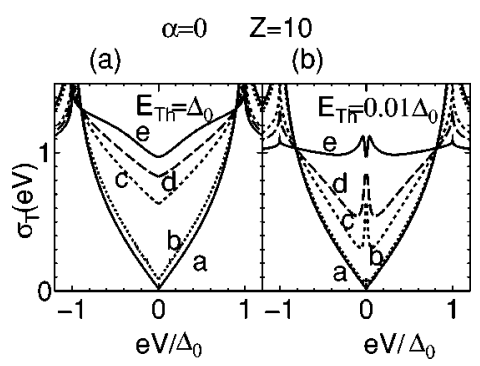

FIG. 1. Normalized conductance $\sigma_{T}(e V)$ for $Z=10$, and $\alpha$ $=0$. (a) $E_{T h}=\Delta_{0}$. (b) $E_{T h}=0.01 \Delta_{0} . a, R_{d} / R_{b}=0 ; b, R_{d} / R_{b}$ $=0.1 ; c, R_{d} / R_{b}=1 ; d, R_{d} / R_{b}=2$; and $e, R_{d} / R_{b}=10$. 


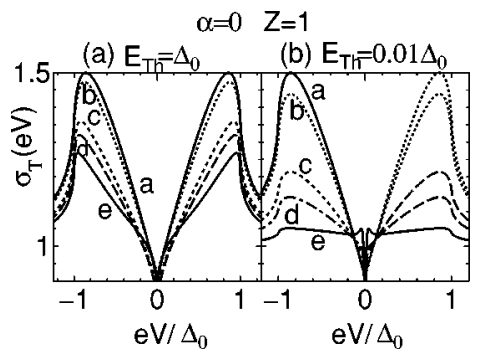

FIG. 2. Normalized conductance $\sigma_{T}(e V)$ for $Z=1$, and $\alpha=0$. (a) $E_{T h}=\Delta_{0}$. (b) $E_{T h}=0.01 \Delta_{0} . a, R_{d} / R_{b}=0 ; b, R_{d} / R_{b}=0.1 ; c$, $R_{d} / R_{b}=1 ; d, R_{d} / R_{b}=2 ; e, R_{d} / R_{b}=10$.

\section{A. $\alpha=0$ without MABS}

Let us first choose $\alpha=0$ where ZBCP due to the MABS is absent. We choose relatively strong barrier $Z=10$ (Fig. 1) for various $R_{d} / R_{b}$. For $E_{T h}=\Delta_{0}$ [see Fig. 1(a)], the magnitude of $\sigma_{T}(e V)$ for $|e V|<\Delta_{0}$ increases with the increase of $R_{d} / R_{b}$. First, the line shape of the voltage-dependent conductance remains to be $\mathrm{V}$ shaped and only the height of the bottom value is enhanced (curves $b$ and $c$ ). The $\mathrm{V}$ shaped line shape originates from the existence of nodes of the $d$-wave pair potential. Then, with a further increase of $R_{d} / R_{b}$, a rounded bottom structure appears (curves $d$ and $e)$. For $E_{T h}=0.01 \Delta_{0}$ [Fig. 1(b)], the magnitude of $\sigma_{T}(e V)$ has a ZBCP once the magnitude of $R_{d} / R_{b}$ deviates slightly from 0 . The order of the magnitude of the $\mathrm{ZBCP}$ width is given by $E_{T h}$ as in the case of $s$-wave junctions. ${ }^{38}$ When the magnitude of $R_{d} / R_{b}$ exceeds unity, the $\sigma_{T}(e V)$ acquires a ZBCD (curve $e$ ). The qualitative features of line shapes of $\sigma_{T}(e V)$ are different from those in $s$-wave junctions (see Figs. 1 and 2 in Ref. 38). It should be remarked that even in the case of $d$-wave junctions we can expect ZBCP by CAR as in the case of $s$-wave junction for $\alpha=0$.

On the other hand, for much more transparent case with $Z=1$ the line shapes of the conductance become quite different. For $E_{T h}=\Delta_{0}$ [Fig. 2(a)], the magnitude of $\sigma_{T}(e V)$ decreases with the increase of the magnitude of $R_{d} / R_{b}$ where the bottom parts of all curves are $\mathrm{V}$ shaped structures. On the other hand, for $E_{T h}=0.01 \Delta_{0}, \sigma_{T}(e V)$ has a ZBCD even for a small magnitude of $R_{d} / R_{b}$. Both for Figs. 2(a) and 2(b), the magnitude of $\sigma_{T}(e V)$ for $|e V|<\Delta_{0}$ decreases with the increase of $R_{d} / R_{b}$. These features are quite different from those shown in Fig. 1.

$$
\alpha=0 \quad Z=0
$$

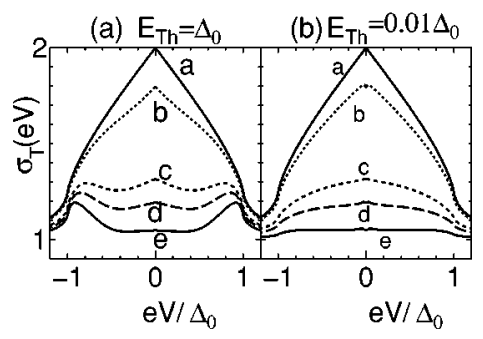

FIG. 3. Normalized conductance $\sigma_{T}(e V)$ for $Z=0$, and $\alpha=0$. (a) $E_{T h}=\Delta_{0}$. (b) $E_{T h}=0.01 \Delta_{0} . a, R_{d} / R_{b}=0 ; b, R_{d} / R_{b}=0.1 ; c$, $R_{d} / R_{b}=1 ; d, R_{d} / R_{b}=2$; and $e, R_{d} / R_{b}=10$.

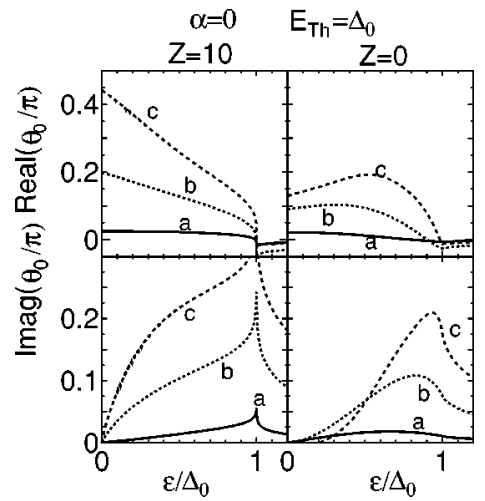

FIG. 4. Real (upper panels) and imaginary parts (lower panels) of $\theta_{0}$ are plotted as a function of $\varepsilon . Z=10$ (left panels) and $Z=0$ (right panels) with $E_{T h} / \Delta_{0}=1$ and $\alpha=0 . a, R_{d} / R_{b}=0.1 ; b$, $R_{d} / R_{b}=1$; and $c, R_{d} / R_{b}=10$.

For transparent limit $Z=0$, the magnitude of $\sigma_{T}(e V)$ decreases with the increase of $R_{d} / R_{b}$ (see Fig. 3). For $E_{T h}$ $=\Delta_{0}, \sigma_{T}(e V)$ has a broad ZBCP for small $R_{d} / R_{b}$. However, with the increase of $R_{d} / R_{b}, \sigma_{T}(e V)$ for $|e V|<\Delta_{0}$ is reduced and becomes nearly constant. For $E_{T h}=0.01 \Delta_{0}$ with $R_{d} / R_{b}$ $=10$, tiny ZBCD appears [curve $e$ of Fig. 3(b)]. As compared to the corresponding case of an $s$-wave junction (see Fig. 4 of Ref. 38), ZBCD is hard to be seen in $d$-wave junctions.

It is interesting to study how various parameters influence the proximity effect. The measure of the proximity effect at the DN/US interface $\theta_{0}$ is plotted for $Z=0$ and $Z=10$ with corresponding parameters in Figs. 1 and 3 (see Figs. 4 and 5). For $R_{d} / R_{b}=0, \theta_{0}=0$ is satisfied for any $E_{T h}$ and $Z$. Besides this fact, at $\varepsilon=0, \theta_{0}$ always becomes a real number. These features are consistent with those in $s$-wave junctions. ${ }^{38}$ First, we study the case of $E_{T h} / \Delta_{0}=1$ (Fig. 4) where the same values of $R_{d} / R_{b}$ are chosen as in Figs. 1 and 3. The real part of $\theta_{0}$ is enhanced with an increase in $R_{d} / R_{b}$ and decreases as a function of $\varepsilon$. At the same time, the imaginary part of $\theta_{0}$ is an increasing function of $\varepsilon$ for $\varepsilon<\Delta_{0}$. Both real and imaginary parts have a sudden change at $\varepsilon$

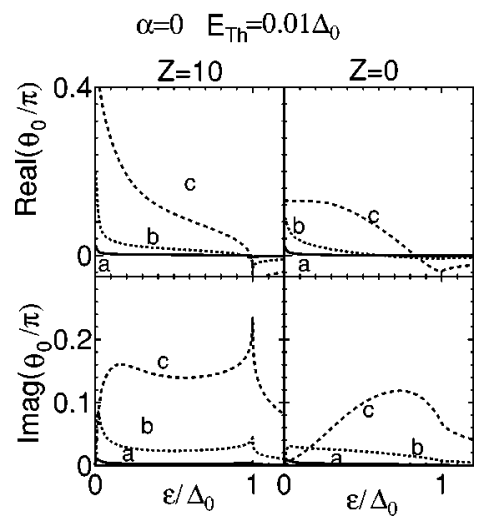

FIG. 5. Real (upper panels) and imaginary parts (lower panels) of $\theta_{0}$ are plotted as a function of $\varepsilon . Z=10$ (left panels) and $Z=0$ (right panels) with $E_{T h} / \Delta_{0}=0.01$ and $\alpha=0 . a, R_{d} / R_{b}=0.1 ; b$, $R_{d} / R_{b}=1$; and $c, R_{d} / R_{b}=10$. 


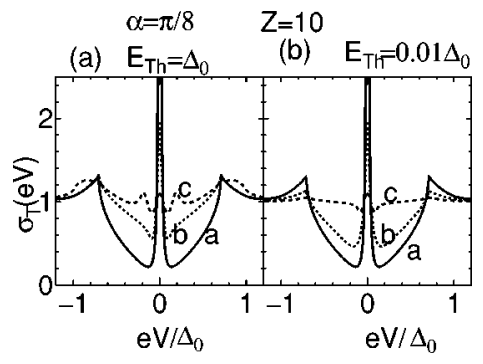

FIG. 6. Normalized conductance $\sigma_{T}(e V)$ for $\alpha=\pi / 8$. (a) $E_{T h}$ $=\Delta_{0}$. (b) $E_{T h}=0.01 \Delta_{0} . a, R_{d} / R_{b}=0 ; b, R_{d} / R_{b}=1$; and $c$, $R_{d} / R_{b}=10$.

$=\Delta_{0}$, where $\Delta_{0}$ is the magnitude of the pair potential felt by quasiparticles with the perpendicular injection. It is remarkable that the magnitude of $\operatorname{Re}(\operatorname{Im})\left(\theta_{0}\right)$ is reduced with the decrease of $Z$. Next, we discuss the line shapes of $\theta_{0}$ for $E_{T h} / \Delta_{0}=0.01 . \operatorname{Re}\left(\theta_{0}\right)$ has a peak at zero voltage and decreases with the increase of $\varepsilon$. $\operatorname{Im}\left(\theta_{0}\right)$ increases sharply from 0 and has a peak at about $\varepsilon \sim E_{T h}$, except for a sufficiently large value of $R_{d}$. These features are consistent with $s$-wave junctions (see Fig. 7 of Ref. 38). Besides this, both real and imaginary parts have a sudden change at $\varepsilon=\Delta_{0}$ as in the case of $E_{T h} / \Delta_{0}=1$. Also in this case, the magnitude of $\operatorname{Re}(\operatorname{Im})\left(\theta_{0}\right)$ is reduced with the decrease of $Z$. This feature can be qualitatively explained as follows. We concentrate on the limiting case $\varepsilon=0$ for simplicity.

The magnitude of $\theta_{0}(0)=\theta_{00}$ is determined by the following equation [see Eq. (32)]:

$$
\begin{gathered}
\frac{\theta_{00}}{R_{d}}=\langle F(\phi)\rangle=\frac{\int_{-\pi / 2}^{\pi / 2} \cos \phi F(\phi) d \phi}{R_{b} \int_{-\pi / 2}^{\pi / 2} \cos \phi T(\phi) d \phi}, \\
F(\phi)=\frac{2 T_{n} \cos \theta_{00} \delta}{2-T_{n}+T_{n} \sin \theta_{00} \delta}, \quad T_{n}=T(\phi)
\end{gathered}
$$

since $f_{+}=f_{-}=\delta$ and $g_{+}=g_{-}=0$ are satisfied with $\delta=1$ $(-1)$ for $-\pi / 4<\phi<\pi / 4 \quad(\pi / 4<|\phi|<\pi / 2)$. The sign change nature of $\delta$ originates from $d$-wave profile of the pair potential. This sign change reduces the magnitude of the right-hand side of Eq. (36), and the resulting $\theta_{00}$ is small. For the case of large magnitude of $Z$, the degree of the reduction due to the sign change of $\delta$, i.e., $F(\phi)$ is not significant. For large magnitude of $Z$ due to the existence of the factor $T_{n}$ $\left(T_{n} \ll 1\right)$ proportional to $\cos ^{2} \phi$, only the small value of $\phi$ can contribute to the integral of numerator where $\delta=1$. This is the reason why the obtained measure of $\theta_{00}$ for $Z=0$ is much smaller than that for $Z=10$.

Although the magnitude of $\theta_{0}$, i.e., the measure of the proximity effect, is enhanced with increasing $R_{d} / R_{b}$, its influence on $\sigma_{T}(e V)$ is different for low and high transparent junctions. In the low transparent junctions, the increase in the magnitude of $\theta_{0}$ by $R_{d} / R_{b}$ can enhance the conductance $\sigma_{T}(e V)$ for $e V \sim 0$ and produce a $\mathrm{ZBCP}$, whereas in high transparent junctions the enhancement of $\theta_{0}$ produces the

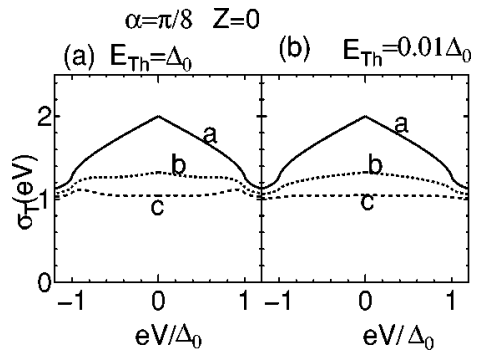

FIG. 7. Normalized conductance $\sigma_{T}(e V)$ for $Z=0$, and $\alpha$ $=\pi / 8$. (a) $E_{T h}=\Delta_{0}$. (b) $E_{T h}=0.01 \Delta_{0} . a, R_{d} / R_{b}=0 ; b, R_{d} / R_{b}$ $=1$; and $c, R_{d} / R_{b}=10$.

ZBCD. However, the amplitude of dip is reduced since the magnitude of $\theta_{00}$ becomes small due to the sign change of $F(\phi)$.

\section{B. $\alpha \neq 0$ with MABS}

In this subsection, we focus on $\sigma_{T}(e V)$ and $\theta_{0}$ for $\alpha \neq 0$ $(0<\alpha<\pi / 4)$. First we focus on $\alpha=\pi / 8$, where MABS is formed for $\pi / 8<|\phi|<3 \pi / 8$. In the low transparent case, i.e., $Z=10, \sigma_{T}(e V)$ has a $\mathrm{ZBCP}$ due to the formation of MABS at the DN/US interface. The height of ZBCP is reduced with the increase of $R_{d} / R_{b}$ (see Fig. 6). Contrary to the corresponding case of $s$-wave junctions (see Fig. 1), $\sigma_{T}(e V)$ is almost independent of $E_{T h}$. For $Z=0$ (see Fig. 7), $\sigma_{T}(e V)$ has a broad ZBCP both for $E_{T h}=\Delta_{0}$ and $E_{T h}$ $=0.01 \Delta_{0}$. With the increase of $R_{d} / R_{b}$, only the magnitude of $\sigma_{T}(e V)$ is reduced and ZBCD does not appear.

It is also interesting to see how $\theta_{0}$ is influenced by various parameters. In Fig. 8, line shapes of $\theta_{0}$ for $E_{T h} / \Delta_{0}=1$ are plotted for various parameters. For $Z=10$, the magnitude of $\operatorname{Re}\left(\theta_{0}\right)$ is drastically suppressed at $\varepsilon \sim 0$ and is an increasing function of $\varepsilon$ contrary to the case of $\alpha=0 . \operatorname{Im}\left(\theta_{0}\right)$ has a peak around $\varepsilon \sim 0.7 \Delta_{0} \sim \Delta_{0} \cos (2 \alpha)$, where $\Delta_{0} \cos (2 \alpha)$ is the magnitude of the pair potential felt by quasiparticles with perpendicular injection. For $Z=0, \varepsilon$ dependence of $\theta_{0}$ is qualitatively similar to the corresponding case of $\alpha=0$ (see Fig. 4), since the role of MABS is not important. For $E_{T h} / \Delta_{0}=0.01$, the magnitude of $\operatorname{Re}\left(\theta_{0}\right)$ at $\varepsilon \sim 0$ is sup-

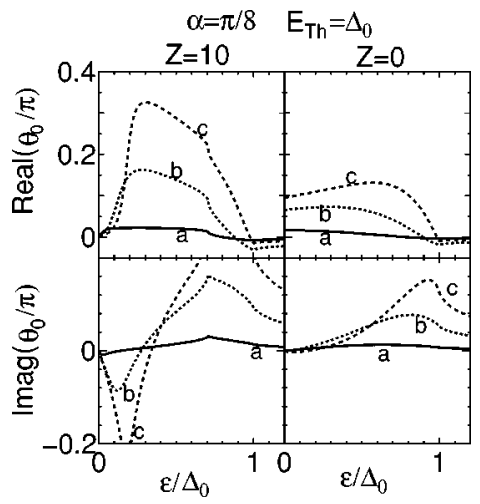

FIG. 8. Real (upper panels) and imaginary parts (lower panels) of $\theta_{0}$ are plotted as a function of $\varepsilon . Z=10$ (left panels) and $Z=0$ (right panels) with $E_{T h} / \Delta_{0}=1$ and $\alpha=\pi / 8 . a, R_{d} / R_{b}=0.1 ; b$, $R_{d} / R_{b}=1$; and $c, R_{d} / R_{b}=10$. 


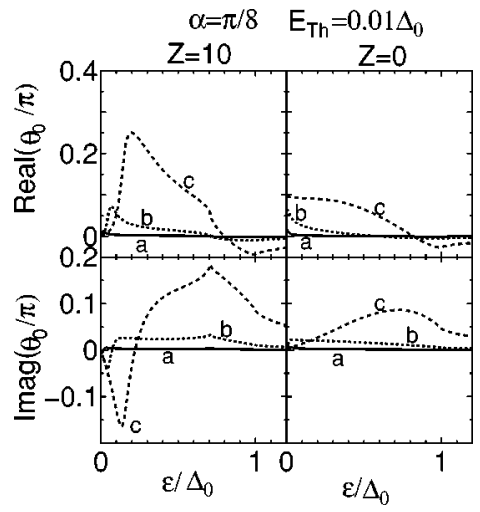

FIG. 9. Real (upper panels) and imaginary parts (lower panels) of $\theta_{0}$ are plotted as a function of $\varepsilon . Z=10$ (left panels) and $Z=0$ (right panels) with $E_{T h} / \Delta_{0}=0.01$ and $\alpha=\pi / 8 . a, R_{d} / R_{b}=0.1 ; b$, $R_{d} / R_{b}=1$; and $c, R_{d} / R_{b}=10$.

pressed (see Fig. 9) for $Z=10 . \operatorname{Im}\left(\theta_{0}\right)$ has a peak around $\varepsilon \sim 0.7 \Delta_{0}$ as in the case of $E_{T h} / \Delta_{0}=1$. On the other hand, for high transparent case, i.e., $Z=0, \operatorname{Re}\left(\theta_{0}\right)$ has a peak at $\varepsilon=0$ and decreases with the increase of $\varepsilon \cdot \operatorname{Im}\left(\theta_{0}\right)$ increases sharply from 0 and has a peak at about $\varepsilon \sim E_{T h}$, except for a sufficiently large value of $R_{d}$. These features are qualitatively consistent with $\alpha=0$ (see Fig. 5).

In order to understand these profiles, we focus on the case with $\varepsilon=0 . \theta_{00}$ is determined by the following relation for $\alpha \neq 0$ :

$$
\begin{gathered}
\frac{\theta_{00}}{R_{d}}=\frac{\langle F(\phi)\rangle}{R_{b}}, \\
F(\phi)= \begin{cases}\frac{2 T(\phi) \cos \theta_{00}}{2-T(\phi)+T(\phi) \sin \theta_{00}}, & 0<|\phi|<\pi / 4-\alpha \\
-2 \tan \theta_{00}, & \pi / 4-\alpha<|\phi|<\pi / 4+\alpha \\
\frac{-2 T(\phi) \cos \theta_{00}}{2-T(\phi)-T(\phi) \sin \theta_{00}}, & \pi / 4+\alpha<|\phi|<\pi / 2 .\end{cases}
\end{gathered}
$$

After simple algebra, we obtain

$$
\begin{gathered}
\frac{\theta_{00}}{R_{d}}=\frac{f_{S 1}+f_{S 2}+f_{S 3}}{R_{b} f_{N}}, \\
f_{S 1}=-2 \sqrt{2} \tan \theta_{00} \sin \alpha \\
f_{S 2}=\int_{0}^{\pi / 4-\alpha} \cos \phi \frac{2 T(\phi) \cos \theta_{00}}{2-T(\phi)+T(\phi) \sin \theta_{00}} d \phi \\
f_{S 3}=-\int_{\pi / 4+\alpha}^{\pi / 2} \cos \phi \frac{2 T(\phi) \cos \theta_{00}}{2-T(\phi)-T(\phi) \sin \theta_{00}} d \phi, \\
f_{N}=\int_{0}^{\pi / 2} T(\phi) \cos \phi d \phi .
\end{gathered}
$$

In the above, $f_{S 1}$ denotes the contribution to $\langle F(\phi)\rangle$ from the unconventional channel with MABS and $f_{S 2}$ and $f_{S 3}$ de-

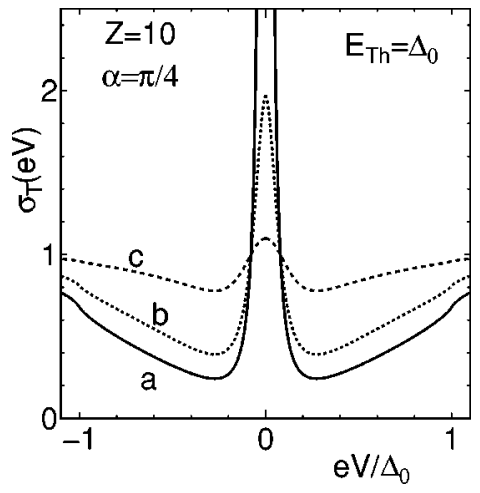

FIG. 10. Normalized conductance $\sigma_{T}(e V)$ for $Z=10, E_{T h} / \Delta_{0}$ $=1$ and $\alpha=\pi / 4$. $a, R_{d} / R_{b}=0 ; b, R_{d} / R_{b}=1$; and $c, R_{d} / R_{b}=10$. In this case, $\sigma_{T}(e V)$ is completely independent of the magnitude of $E_{T h}$.

note that from the conventional channel without MABS. For low transparent case, $T(\phi) \ll 1$, the magnitude of $f_{S 1}$ dominates over those of $f_{S 2}$ and $f_{S 3}$ and $\theta_{00}$ is determined by

$$
\frac{\theta_{00}}{R_{d}} \sim \frac{-2 \sqrt{2} \tan \theta_{00} \sin \alpha}{R_{b} f_{N}},
$$

then the resulting $\theta_{00}$ is reduced to be almost zero. As seen from this, MABS and proximity effect strongly compete each other. While for high transparent limit, $T(\phi)=1$, the magnitude of $f_{S 1}$ becomes the same order as those of $f_{S 2}$ and $f_{S 3}$. Then from the contribution by conventional channel, i.e., $f_{S 2}$ and $f_{S 3}$, the magnitude of $\theta_{00}$ is much larger than that for $Z=10$.

For $\alpha=\pi / 4$, where $f_{S 2}=f_{S 3}=0$ is satisfied, only the unconventional channel $f_{S 1}$ can contribute to $\langle F(\phi)\rangle$. Not only $\theta_{00}$ but $\theta_{0}$ for any $\varepsilon$ is exactly zero. Then the total resistance $R$ can be given by ${ }^{108}$

$$
R=\frac{R_{b}}{\left\langle I_{b 0}\right\rangle}+R_{d}=R_{R_{d}=0}+R_{d}
$$

and the resulting $\sigma_{T}(e V)$ is given by

$$
\sigma_{T}(e V)=\frac{R_{d}+R_{b}}{R_{R_{d}=0}+R_{d}} .
$$

One of the typical examples is plotted in Fig. 10.

The effect of $R_{d}$ is significant for the resulting $\sigma_{T}(e V)$. For the actual quantitative comparison with tunneling experiments, we must take into account the effect of $R_{d}$.

\section{C. $\alpha$ dependence of zero-voltage conductance}

Finally, we study the dependence of $\sigma_{T}(0)$ on the angle $\alpha$. In this case, $\sigma_{T}(0)$ is independent of $E_{T h}$. For all situations shown in Figs. $11-13, \theta_{00}=0$ is satisfied for $\alpha=\pi / 4$, due to the complete absence of proximity effect where only the unconventional channel with MABS exists. For $Z=0$, 


\section{$Z=0$}

(a)

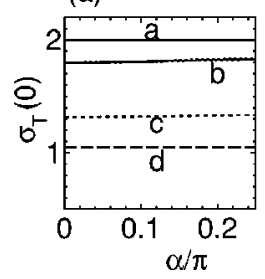

(b)

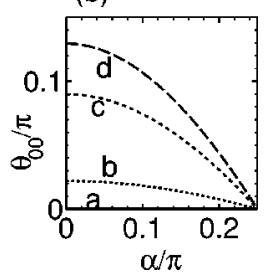

FIG. 11. Normalized conductance at zero voltage $\sigma_{T}(0)$ for various $\alpha$ with $Z=0$. In this case $\sigma_{T}(0)$ is independent of $E_{T h} . a$, $R_{d} / R_{b}=0 ; b, R_{d} / R_{b}=0.1 ; c, R_{d} / R_{b}=1 ;$ and $d, R_{d} / R_{b}=10$.

$\sigma_{T}(0)$ is almost constant with the change of $\alpha$ although $\theta_{00}$ is a decreasing function of $\alpha$ (see Fig. 11).

For $Z=1, \sigma_{T}(0)$ is an increasing function of $\alpha$, while $\theta_{00}$ is a decreasing function (see Fig. 12). For $Z=10, \sigma_{T}(0)$ is enhanced much more rapidly as compared to the case for $Z$ $=1$, while with the increase of $R_{d} / R_{b}, \sigma_{T}(0)$ becomes nearly constant (see Fig. 13). As seen from Figs. 12 and 13, the influence of $R_{d} / R_{b}$ on the $\sigma_{T}(0)$ is significantly important.

In order to understand these features we look at $I_{b 0}$ in detail. In general, for $\varepsilon=0,\left\langle I_{b 0}\right\rangle$ can be expressed by

$$
\begin{gathered}
\left\langle I_{b 0}\right\rangle=\frac{I_{b 1}+I_{b 2}+I_{b 3}}{I_{n}} \\
I_{b 1}=2 \sqrt{2} \sin \alpha \sec ^{2} \theta_{00} \\
I_{b 2}=\int_{0}^{\pi / 4-\alpha} \cos \phi \frac{2 T(\phi)\left[T(\phi)+\{2-T(\phi)\} \sin \theta_{00}\right]}{\left|2-T(\phi)+T(\phi) \sin \theta_{00}\right|^{2}} \\
I_{b 3}=\int_{\pi / 4+\alpha}^{\pi / 2} \cos \phi \frac{2 T(\phi)\left[T(\phi)-\{2-T(\phi)\} \sin \theta_{00}\right]}{\left|2-T(\phi)-T(\phi) \sin \theta_{00}\right|^{2}} \\
I_{n}=\int_{0}^{\pi / 2} T(\phi) \cos \phi d \phi .
\end{gathered}
$$

$I_{b 1}$ denotes the contribution from the unconventional channel while $I_{b 2}$ and $I_{b 3}$ denote those from the conventional channel. For $Z=0$, the integral can be performed analytically. For $\alpha=0,\left\langle I_{b 0}\right\rangle$ becomes $2 \sec ^{2} \theta_{00}\left[1-(\sqrt{2}-1) \sin \theta_{00}\right]$ for $\alpha$ $=0$ and $2 \sec ^{2} \theta_{00}$ for $\alpha=\pi / 4$. Since the order of $\theta_{00}$ is at

\section{$Z=1$}
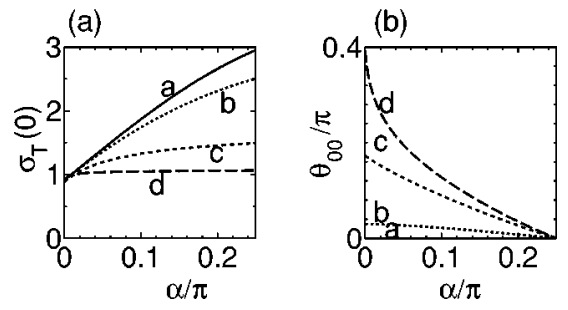

FIG. 12. Normalized conductance at zero voltage $\sigma_{T}(0)$ for various $\alpha$ with $Z=1$. In this case $\sigma_{T}(0)$ is independent of $E_{T h} . a$, $R_{d} / R_{b}=0 ; b, R_{d} / R_{b}=0.1 ; c, R_{d} / R_{b}=1 ;$ and $d, R_{d} / R_{b}=10$.
$Z=10$
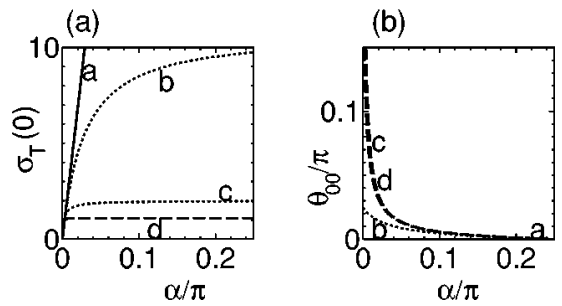

FIG. 13. Normalized conductance at zero voltage $\sigma_{T}(0)$ for various $\alpha$ with $Z=10$. In this case $\sigma_{T}(0)$ is independent of $E_{T h} . a$, $R_{d} / R_{b}=0 ; b, R_{d} / R_{b}=0.1 ; c, R_{d} / R_{b}=1$; and $d, R_{d} / R_{b}=10$.

most 0.1 as seen from Fig. 11(b), the difference between the two cases is small. Then we can expect that $\sigma_{T}(0)$ is almost constant as a function of $\alpha$. On the other hand, for $Z \neq 0$, the contribution from the unconventional channel becomes significant and the resulting $\left\langle I_{b 0}\right\rangle$ can be approximated to be

$$
\left\langle I_{b 0}\right\rangle \sim 2 \sqrt{2} \sin \alpha \sec ^{2} \theta_{00} / I_{n} .
$$

It is an increasing function of $\alpha$, and the resulting $\sigma_{T}(0)$ is also an increasing function of $\alpha$ (see Figs. 12 and 13).

\section{CONCLUSIONS}

In the present paper, detailed theoretical investigation of the voltage-dependent conductance of DN/US junctions is presented. We have provided the detailed derivation of the expression for the matrix current presented in our previous paper. ${ }^{108}$ For the reader's convenience, we explicitly present the retarded and the Keldysh parts of the matrix current for the case when the US has a singlet parity. Applying these expressions to $\mathrm{DN} / d$-wave junctions, we have obtained the following main results.

(1) There are two kinds of ZBCP, i.e., ZBCP due to the CAR by proximity effect in $\mathrm{DN}$ and that due to the formation of MABS at interfaces of $d$-wave superconductors. ZBCP frequently appears in the line shapes of $\sigma_{T}(e V)$. For low transparent junctions with small Thouless energy $E_{T h}$ we always expect ZBCP independent of $\alpha$.

(2) The nature of ZBCP due to the MABS and that by CAR is significantly different. The corresponding $\sigma_{T}(0)$ for the former case can take arbitrary values exceeding unity. On the other hand, $\sigma_{T}(0)$ for the latter case never exceeds unity. The width of the ZBCP in the former case is determined by the transparency of the junction while the width for the latter case is determined by the Thouless energy. These two ZBCP's compete each other since the proximity effect and the existence of MABS are incompatible in singlet junctions. $^{108}$

(3) For the extreme case, $\alpha=\pi / 4$, where the proximity effect is absent and the CAR is canceled, $\sigma_{T}(e V)$ is given by $\sigma_{T}(e V)=\left(R_{b}+R_{d}\right) /\left(R_{R_{d}=0}+R_{d}\right)$ with the resistance at the interface $R_{b}$.

(4) Only when $\alpha=0$ MABS is absent for $R_{d}=0$. Then CAR influences significantly $\sigma_{T}(e V)$, similarly to the case of an $s$-wave junction. When the transparency of the junction is sufficiently low, $\sigma_{T}(e V)$ for $|e V|<\Delta_{0}$ is enhanced with 
the increase of $R_{d}$ due to the enhancement of the proximity effect assisted by CAR. The ZBCP becomes prominent for $E_{T h} \ll \Delta_{0}$ and $R_{d} / R_{b}<1$. In such a case, with a further increase of $R_{d} / R_{b}$ the ZBCP changes into a ZBCD.

(5) We have clarified various line shapes of the conductance including ZBCP. The obtained results serve as an important guide to analyze the actual experimental data of the tunneling spectra of high- $T_{C}$ cuprate junctions. We want to stress that the height of ZBCP is strongly suppressed by the existence of $\mathrm{DN}$ and the resulting $\sigma_{T}(0)$ is not so high as obtained in the ballistic regime. ${ }^{40}$ In the actual fit of the experimental data, we strongly hope to take into account the effect of $R_{d}$. When the transparency of the junction is low and $\alpha \neq 0$, the contribution of unconventional channel becomes important and that from conventional channel is negligible. In such a case without solving Usadel equation $\sigma_{T}(e V)$ can be simply approximated by

$$
\begin{gathered}
\sigma_{T}(e V)=\frac{R_{d}+R_{b}}{R_{R_{d}=0}+R_{d}}, \quad R_{R_{d}=0}=\frac{R_{b}}{\left\langle I_{b 0}\right\rangle}, \\
\left\langle I_{b 0}\right\rangle=\frac{\int_{-\pi / 2}^{\pi / 2} \cos \phi I_{b 0} d \phi}{\int_{-\pi / 2}^{\pi / 2} \cos \phi T(\phi) d \phi}, \\
I_{b 0}=\frac{T(\phi)\left\{1+T(\phi)\left|\Gamma_{+}\right|^{2}+[T(\phi)-1]\left|\Gamma_{+} \Gamma_{-}\right|^{2}\right\}}{\left|1+[T(\phi)-1] \Gamma_{+} \Gamma_{-}\right|^{2}}, \\
\Gamma_{+}=\frac{\Delta_{+}}{\varepsilon+\sqrt{\varepsilon^{2}-\Delta_{+}^{2}}}, \quad \Gamma_{-}=\frac{\Delta_{-}}{\varepsilon+\sqrt{\varepsilon^{2}-\Delta_{-}^{2}}}, \\
R_{b}=\frac{h}{2 e^{2}} \frac{2}{\int_{-\pi / 2}^{\pi / 2}} d \phi T(\phi) \cos \phi
\end{gathered}
$$

with $\Delta_{ \pm}=\Delta_{0} \cos [2(\phi \bar{\mp} \alpha)]$ and $\varepsilon=e V$. This expression is a convenient one for the fit of the experimental data. However, for the quantitative discussions including much more general cases, one must solve the Usadel equation as was done in the present paper. It is an interesting future problem to compare the present results with experiments since recent experimental results show the existence of mesoscopic coherence in high $T_{C}$ cuprate junctions. ${ }^{110}$

There are several problems which are not discussed in the present paper. In the present study, we have focused on N/S junctions. The extension of the circuit theory to long diffusive $\mathrm{S} / \mathrm{N} / \mathrm{S}$ junctions has been performed by Bezuglyi et al. ${ }^{36}$ In $\mathrm{S} / \mathrm{N} / \mathrm{S}$ junctions, the mechanism of multiple Andreev reflections produces the subharmonic gap structures on $I-V$ curves ${ }^{111-117}$ and the situation becomes much more complex as compared to N/S junctions. Moreover, in S/N/S junctions with unconventional superconductors, MABS leads to the anomalous current-phase relation and temperature dependence of the Josephson current. ${ }^{89}$ An interesting problem is an extension of the circuit theory to $\mathrm{S} / \mathrm{N} / \mathrm{S}$ junctions with unconventional superconductors.

There are two kinds of ZBCP's considered in the present paper. We expect that the response to the magnetic field should be significantly different in these two cases. The $\mathrm{ZBCP}$ originating from MABS is rather robust against the magnetic field while that from CAR is much more sensitive. We want to clarify this feature in actual calculations.

In the present paper, since we follow the quasiclassical Green's function formalism, the impurity scattering is taken into account within the self-consistent Born approximation. It is a challenging problem to study the weak localization effects.

\section{ACKNOWLEDGMENTS}

The authors appreciate useful and fruitful discussions with J. Inoue, H. Itoh, Y. Asano, and I. Shigeta. This work was supported by the Core Research for Evolutional Science and Technology (CREST) of the Japan Science and Technology Corporation (JST). The computational aspect of this work was performed at the facilities of the Supercomputer Center, Institute for Solid State Physics, University of Tokyo and the Computer Center.

\section{APPENDIX}

The matrix current is expressed as

$$
\check{I}=\frac{2 e^{2}}{h} \operatorname{Tr}_{n, \sigma}\left[\bar{\Sigma}^{z} \bar{g}_{1}\right]=\frac{2 e^{2}}{h} \operatorname{Tr}_{n, \sigma}\left[\bar{\Sigma}^{z} \bar{g}_{2}\right] .
$$

To find the matrix current, we have to evaluate $\bar{g}_{1(2)}$. For this purpose, we shall consider the behavior of $\check{G}_{n}^{\sigma \sigma^{\prime}}\left(x, x^{\prime}\right)$ in the isotropization zone in DN side $\left(-L_{1}<x, x^{\prime}<-L_{2}\right)$ and in the ballistic zone of right side $\left(x, x^{\prime}>0\right)$. In the isotropic zone, since the effect of impurity scattering is dominant and $\check{H}$ can be approximated to be $-\check{\Sigma}_{\text {imp }}(x)$ $=\check{G}_{1} /\left(2 \tau_{\text {imp }}\right)$ for $x<0 . \check{G}_{1}$ is the Keldysh-Nambu Green's function in DN at $x=-L_{1}$ with $\xi_{1} \gg L_{1} \gg v \tau_{i m p}$ and $\xi_{1} \gg L_{1}$ $-L_{2} \gg v \tau_{\text {imp }}$ where $\xi_{1}=\sqrt{D / 2 \pi T}$ is the coherence length of the Green's function in DN. Due to this condition, $\check{G}_{1}$ can be approximated to be $\check{G}_{1}=\check{G}_{N}\left(-L_{1}\right) \sim \check{G}_{N}\left(0_{-}\right)$, where $\check{G}_{N}(x)$ obeys the Usadel equation in DN. The Green's function $\check{G}_{n}^{\sigma \sigma^{\prime}}\left(x, x^{\prime}\right)$ is expressed by

$$
\begin{gathered}
\check{G}_{n}^{\sigma \sigma^{\prime}}\left(x, x^{\prime}\right)=\bar{P}(x)\left[\bar{g}_{1}+\operatorname{sgn}\left(x-x^{\prime}\right) \overline{\Sigma^{z}}\right] \bar{P}\left(-x^{\prime}\right), \\
\bar{P}(x)=\frac{1}{2 \sqrt{2 v_{n} i}}\left\{\exp \left[x /\left(2 v_{n} \tau_{i m p}\right)\right]\left(\overline{1}-\bar{\Sigma}^{z} \bar{G}_{1}\right)\right. \\
\left.+\exp \left[-x /\left(2 v_{n} \tau_{i m p}\right)\right]\left(\overline{1}+\bar{\Sigma}^{z} \bar{G}_{1}\right)\right\}
\end{gathered}
$$

with

$$
\bar{G}_{1}=\left(\begin{array}{cc}
\check{G}_{1} & 0 \\
0 & \check{G}_{1}
\end{array}\right), \quad \bar{\Sigma}^{z}=\left(\begin{array}{cc}
\check{1} & 0 \\
0 & -\check{1}
\end{array}\right) .
$$


To ensure that $\check{G}_{n}^{\sigma \sigma^{\prime}}\left(x, x^{\prime}\right)$ does not grow with decreasing $x$ and $x^{\prime}$ in the isotropic zone in $\mathrm{DN}$, we shall require

$$
\left(\bar{\Sigma}^{z}+\bar{G}_{1}\right)\left(\bar{\Sigma}^{z}-\bar{g}_{1}\right)=0
$$

$$
\left(\bar{\Sigma}^{z}+\bar{g}_{1}\right)\left(\bar{\Sigma}^{z}-\bar{G}_{1}\right)=0 .
$$

On the other hand, in the US side $(x>0)$ it is not simple to obtain $\check{G}_{n}^{\sigma \sigma^{\prime}}\left(x, x^{\prime}\right)$ since it has directional dependence. $\check{G}_{n}^{\sigma \sigma^{\prime}}\left(x, x^{\prime}\right)$ is given by

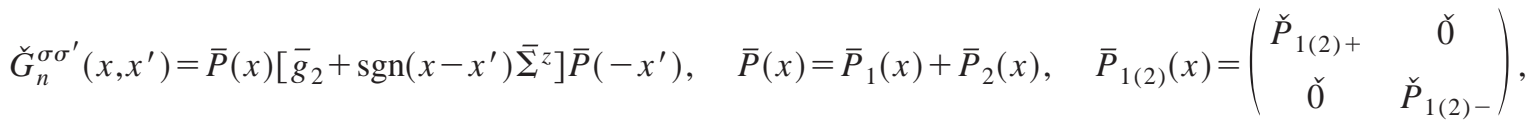

$$
\begin{aligned}
& \check{P}_{1 \pm}(x)=\frac{1}{2 \sqrt{2 v_{n} i}}\left(\begin{array}{cc}
\gamma_{ \pm}\left(1 \mp \hat{R}_{2 \pm}\right) & f_{0}\left[\gamma_{ \pm}\left(1 \mp \hat{R}_{2 \pm}\right)-\gamma_{ \pm}^{*}\left(1 \mp \hat{A}_{2 \pm}\right)\right] \\
0 & \gamma_{ \pm}^{*}\left(1 \mp \hat{A}_{2 \pm}\right)
\end{array}\right), \\
& \check{P}_{2 \pm}(x)=\frac{1}{2 \sqrt{2 v_{n} i}}\left(\begin{array}{cc}
\bar{\gamma}_{ \pm}\left(1 \pm \hat{R}_{2 \pm}\right) & f_{0}\left[\bar{\gamma}_{ \pm}\left(1 \pm \hat{R}_{2 \pm}\right)-\bar{\gamma}_{ \pm}^{*}\left(1 \pm \hat{A}_{2 \pm}\right)\right] \\
0 & \bar{\gamma}_{ \pm}^{*}\left(1 \pm \hat{A}_{2 \pm}\right)
\end{array}\right)
\end{aligned}
$$

with

$$
\gamma_{ \pm}=\exp \left[-i \frac{\sqrt{(\varepsilon+i \delta)^{2}-\Delta_{ \pm}^{2}}}{\hbar v_{F x}} x\right]
$$

and

$$
\bar{\gamma}_{ \pm}=\exp \left[i \frac{\sqrt{(\varepsilon+i \delta)^{2}-\Delta_{ \pm}^{2}}}{\hbar v_{F x}} x\right]
$$

In the above, $\hat{R}_{2 \pm}$ and $\hat{A}_{2 \pm}$ are retarded and advanced components of Keldysh-Nambu Green's function $\check{G}_{2 \pm}$ at the interface of US where \pm denotes the direction of motion along $x$ axis. $\check{G}_{2+}$ and $\check{G}_{2-}$ are given by $\check{G}_{2 \pm}=\check{G}_{S \pm}\left(0_{+}\right)$where $\check{G}_{S \pm}(x)$ is a quasiclassical Green's function in US. It does depend on the direction of motion $\sigma$. Here we neglect the spatial dependence for simplicity and we assume $\check{G}_{2 \pm}$ $=\check{G}_{S \pm}(x)=\check{G}_{S_{ \pm}}(\infty)$. Since $\gamma_{ \pm}$and $\gamma_{ \pm}^{*}$ are glowing functions with the increase of $x$, the term that includes this component should be eliminated by multiplying $\left[\bar{g}_{2}+\operatorname{sgn}(x\right.$ $\left.\left.-x^{\prime}\right) \bar{\Sigma}^{z}\right]$. For convenience, we denote

$$
\bar{g}_{2}+\operatorname{sgn}\left(x-x^{\prime}\right) \bar{\Sigma}^{z}=\left(\begin{array}{cc}
\check{A} & \check{C} \\
\check{B} & \check{D}
\end{array}\right) .
$$

In order to eliminate the divergence terms with the increase of $x(x>0)$,

$$
\begin{gathered}
\left(\begin{array}{cc}
\gamma_{+}\left(1-\hat{R}_{2+}\right) & f_{0}\left[\gamma_{+}\left(1-\hat{R}_{2+}\right)-\gamma_{+}^{*}\left(1-\hat{A}_{2+}\right)\right] \\
0 & \gamma_{+}^{*}\left(1-\hat{A}_{2+}\right)
\end{array}\right)\left(\begin{array}{cc}
\hat{a} & \hat{c} \\
\hat{b} & \hat{d}
\end{array}\right) \\
=0, \quad \check{A}=\left(\begin{array}{cc}
\hat{a} & \hat{c} \\
\hat{b} & \hat{d}
\end{array}\right)
\end{gathered}
$$

should be satisfied. Then following four equations must be satisfied for any $x$ :

$$
\begin{gathered}
\gamma_{+}\left(1-\hat{R}_{2+}\right) \hat{a}+f_{0}\left[\gamma_{+}\left(1-\hat{R}_{2+}\right)-\gamma_{+}^{*}\left(1-\hat{A}_{2+}\right)\right] \hat{b}=0, \\
\gamma_{+}^{*}\left(1-\hat{A}_{2+}\right) \hat{b}=0, \\
\gamma_{+}^{*}\left(1-\hat{A}_{2+}\right) \hat{d}=0, \\
\gamma_{+}\left(1-\hat{R}_{2+}\right) \hat{c}+f_{0}\left[\gamma_{+}\left(1-\hat{R}_{2+}\right)-\gamma_{+}^{*}\left(1-\hat{A}_{2+}\right)\right] \hat{d}=0 .
\end{gathered}
$$

Thus we obtain

$$
\left(\begin{array}{cc}
\left(1-\hat{R}_{2+}\right) & \left.f_{0}\left[-\hat{R}_{2+}+\hat{A}_{2+}\right)\right] \\
0 & \left(1-\hat{A}_{2+}\right)
\end{array}\right)\left(\begin{array}{cc}
\hat{a} & \hat{c} \\
\hat{b} & \hat{d}
\end{array}\right)=0 .
$$

Applying the similar discussions for other components, we also obtain the following equations:

$$
\left(\begin{array}{cc}
\check{E}_{+} & 0 \\
0 & \check{E}_{-}
\end{array}\right)\left(\begin{array}{cc}
\check{A} & \check{C} \\
\check{B} & \check{D}
\end{array}\right)=0
$$

with

$$
\check{E}_{ \pm}=\left(\begin{array}{cc}
\left(1-\hat{R}_{2 \pm}\right) & \left.f_{0}\left[-\hat{R}_{2 \pm}+\hat{A}_{2 \pm}\right)\right] \\
0 & \left(1-\hat{A}_{2 \pm}\right)
\end{array}\right) .
$$

The resulting equation is identical to Eq. (30) of Ref. 37.

$$
\left(\bar{\Sigma}^{z}-\bar{G}_{2}\right)\left(\bar{g}_{2}+\bar{\Sigma}^{z}\right)=0 .
$$

Applying similar discussion for the case with increasing $x^{\prime}$ $\left(x^{\prime}>0\right)$, we can also obtain

$$
\left(\bar{g}_{2}-\bar{\Sigma}^{z}\right)\left(\bar{\Sigma}^{z}+\bar{G}_{2}\right)=0
$$


To find explicit expression of $\bar{g}_{2}$, we multiply Eq. (A4) by $\bar{M}^{\dagger}$ from the left and by $\bar{M}$ from the right. By introducing the matrix $\bar{Q}=\bar{M}^{\dagger} \bar{M}$, we derive

$$
\bar{g}_{2}=\left(\bar{Q} \bar{G}_{2}+\bar{G}_{1}\right)^{-1}\left\{2 \bar{Q}+\left(\bar{G}_{1}-\bar{Q} \bar{G}_{2}\right) \bar{\Sigma}^{z}\right\}
$$

with

$$
\bar{G}_{2}=\left(\begin{array}{cc}
\check{G}_{2+} & 0 \\
0 & \check{G}_{2-}
\end{array}\right) .
$$

To evaluate matrix current $\check{I}$, we rewrite $\bar{g}_{2}$ in the basis composed of eigenvectors of $\bar{Q}$ and Keldysh-Nambu indices. For each eigenvector $\vec{c}_{n}$ with eigenvalue $q_{n}>1$, the vector $\bar{\Sigma}^{z} \vec{c}_{n}$ is also an eigenvector of $\bar{Q}$ with eigenvalue $q_{n}^{-1}$,

$$
\bar{Q}=\left(\begin{array}{cc}
q_{n} & 0 \\
0 & q_{n}^{-1}
\end{array}\right), \quad \bar{\Sigma} z=\left(\begin{array}{cc}
0 & \check{1} \\
\check{1} & 0
\end{array}\right) .
$$

The resulting $\bar{g}_{2}$ is given by

$$
\begin{aligned}
\bar{g}_{2}= & \left(\begin{array}{cc}
q_{n} \check{H}_{+}+\check{G}_{1} & q_{n} \check{H}_{-} \\
q_{n}^{-1} \check{H}_{-} & q_{n}^{-1} \check{H}_{+}+\check{G}_{1}
\end{array}\right)^{-1} \\
& \times\left(\begin{array}{cc}
q_{n}\left(2-\check{H}_{-}\right) & \check{G}_{1}-q_{n} \check{H}_{+} \\
\check{G}_{1}-q_{n}^{-1} \check{H}_{+} & q_{n}^{-1}\left(2-\check{H}_{-}\right)
\end{array}\right)
\end{aligned}
$$

with $\check{H}_{ \pm}=\left(\check{G}_{2+} \pm \check{G}_{2-}\right) / 2$. Then the matrix current $\check{I}$ can be expressed as

$$
\begin{aligned}
\check{I}=\frac{2 e^{2}}{h} \sum_{n} \check{I}_{n 0}, \\
\check{I}_{n 0}=\left[\check{H}_{-}-\left(q_{n} \check{H}_{+}+\check{G}_{1}\right) \check{H}_{-}^{-1}\left(\check{H}_{+} / q_{n}+\check{G}_{1}\right)\right]^{-1}\left(2-\check{H}_{-}\right) \\
+\left[\check{H}_{-}-\left(\check{H}_{+} / q_{n}+\check{G}_{1}\right) \check{H}_{-}^{-1}\left(q_{n} \check{H}_{+}+\check{G}_{1}\right)\right]^{-1}\left(2-\check{H}_{-}\right) \\
+\left[\left(\check{H}_{+} / q_{n}+\check{G}_{1}\right)-\check{H}_{-}\left(q_{n} \check{H}_{+}+\check{G}_{1}\right)^{-1} \check{H}_{-}\right]^{-1} \\
\times\left(\check{G}_{1}-\check{H}_{+} / q_{n}\right)+\left[\left(q_{n} \check{H}_{+}+\check{G}_{1}\right)\right. \\
\left.-\check{\check{H}}_{-}\left(\check{H}_{+} / q_{n}+\check{G}_{1}\right)^{-1} \check{H}_{-}\right]^{-1}\left(\check{G}_{1}-q_{n} \check{H}_{+}\right) . \quad \text { (A11) }
\end{aligned}
$$

Applying the following identity:

$$
\check{H}_{+}^{2}+\check{H}_{-}^{2}=\check{1}, \quad \check{H}_{+} \check{H}_{-}+\check{H}_{-} \check{H}_{+}=0
$$

$\check{I}_{n 0}$ can be written as follows:

$$
\begin{aligned}
\check{I}_{n 0}= & \left(\left[\check{G}_{1}, \check{H}_{-}^{-1}\right] q_{n}-\check{H}_{-}^{-1} \check{H}_{+}+\check{G}_{1} \check{H}_{-}^{-1} \check{H}_{+} \check{G}_{1} q_{n}^{2}\right)^{-1}\left[\left(\check{G}_{1}\right.\right. \\
& \left.\left.-\check{H}_{-}^{-1} \check{G}_{1}\right) q_{n}+\check{G}_{1} \check{H}_{-}^{-1} \check{H}_{+} \check{G}_{1} q_{n}^{2}\right]+\left(\left[\check{G}_{1}, \check{H}_{-}^{-1}\right] / q_{n}\right. \\
& \left.-\check{H}_{-}^{-1} \check{H}_{+} / q_{n}^{2}+\check{G}_{1} \check{H}_{-}^{-1} \check{H}_{+} \check{G}_{1}\right)^{-1}\left[\left(\check{G}_{1}-\check{G}_{1} \check{H}_{-}^{-1}\right) / q_{n}\right. \\
& \left.+\check{H}_{-}^{-1} \check{H}_{+} / q_{n}^{2}\right]+\left(\left[\check{G}_{1}, \check{H}_{-}^{-1}\right] / q_{n}-\check{H}_{-}^{-1} \check{H}_{+}\right. \\
& \left.+\check{G}_{1} \check{H}_{-}^{-1} \check{H}_{+} \check{G}_{1} / q_{n}^{2}\right)^{-1}\left[\left(\check{G}_{1}-\check{H}_{-}^{-1} \check{G}_{1}\right) / q_{n}\right. \\
& \left.+\check{G}_{1} \check{H}_{-}^{-1} \check{H}_{+} \check{G}_{1} / q_{n}^{2}\right]+\left(\left[\check{G}_{1}, \check{H}_{-}^{-1}\right] q_{n}-\check{H}_{-}^{-1} \check{H}_{+} q_{n}^{2}\right. \\
& \left.+\check{G}_{1} \check{H}_{-}^{-1} \check{H}_{+} \check{G}_{1}\right)^{-1}\left[\left(\check{G}_{1}-\check{G}_{1} \check{H}_{-}^{-1}\right) q_{n}+\check{H}_{-}^{-1} \check{H}_{+} q_{n}^{2}\right] .
\end{aligned}
$$

The eigenvalue $q_{n}$ and the transparency of the junction $T_{n}$ satisfy the following relations:

$$
4 q_{n} /\left(1+q_{n}\right)^{2}=T_{n}, \quad\left(1+q_{n}^{2}\right) /\left(1+q_{n}\right)^{2}=\left(2-T_{n}\right) / 2 .
$$

We introduce

$$
T_{1 n}=\frac{T_{n}}{2-T_{n}+2 \sqrt{1-T_{n}}}
$$

and the resulting $\check{I}_{n 0}$ becomes

$$
\begin{aligned}
\check{I}_{n 0}= & -\check{D}^{-1}\left\{T_{1 n}\left(2 \check{G}_{1}-\left[\check{H}_{-}^{-1}, \check{G}_{1}\right]_{+}\right)\right. \\
& \left.+\check{H}_{-}^{-1} \check{H}_{+}+T_{1 n}^{2} \check{G}_{1} \check{H}_{-}^{-1} \check{H}_{+} \check{G}_{1}\right\} \\
& +\check{G}_{1} \check{D}^{-1} \check{G}_{1}\left\{T_{1 n}\left(2 \check{G}_{1}-\left[\check{H}_{-}^{-1}, \check{G}_{1}\right]_{+}\right)\right. \\
& \left.+T_{1 n}^{2} \check{H}_{-}^{-1} \check{H}_{+}+\check{G}_{1} \check{H}_{-}^{-1} \check{H}_{+} \check{G}_{1}\right\}
\end{aligned}
$$

with

$$
\check{D}=-T_{1 n}\left[\check{G}_{1}, \check{H}_{-}^{-1}\right]+\check{H}_{-}^{-1} \check{H}_{+}-T_{1 n}^{2} \check{G}_{1} \check{H}_{-}^{-1} \check{H}_{+} \check{G}_{1},
$$

$\check{I}_{n 0}$ is also represented as follows: ${ }^{108}$

$$
\check{I}_{n 0}=2\left[\check{G}_{1}, \check{B}_{n}\right]
$$

with

$$
\begin{gathered}
\check{B}_{n}=\check{B}_{n b}^{-1}\left[T_{1 n}\left(1-\check{H}_{-}^{-1}\right)+T_{1 n}^{2} \check{G}_{1} \check{H}_{-}^{-1} \check{H}_{+}\right], \\
\check{B}_{n b}=\left(-T_{1 n}\left[\check{G}_{1}, \check{H}_{-}^{-1}\right]+\check{H}_{-}^{-1} \check{H}_{+}-T_{1 n}^{2} \check{G}_{1} \check{H}_{-}^{-1} \check{H}_{+} \check{G}_{1}\right) .
\end{gathered}
$$

${ }^{1}$ A.F. Andreev, Zh. Eksp. Teor. Fiz. 46, 1823 (1964) [Sov. Phys. JETP 19, 1228 (1964)].

${ }^{2}$ F.W.J. Hekking and Yu.V. Nazarov, Phys. Rev. Lett. 71, 1625 (1993).

${ }^{3}$ F. Giazotto, P. Pingue, F. Beltram, M. Lazzarino, D. Orani, S. Rubini, and A. Franciosi, Phys. Rev. Lett. 87, 216808 (2001).

${ }^{4}$ T.M. Klapwijk, Physica B 197, 481 (1994).

${ }^{5}$ A. Kastalsky, A.W. Kleinsasser, L.H. Greene, R. Bhat, F.P. Mil- liken, and J.P. Harbison, Phys. Rev. Lett. 67, 3026 (1991).

${ }^{6}$ C. Nguyen, H. Kroemer, and E.L. Hu, Phys. Rev. Lett. 69, 2847 (1992).

${ }^{7}$ B.J. van Wees, P. de Vries, P. Magnee, and T.M. Klapwijk, Phys. Rev. Lett. 69, 510 (1992).

${ }^{8}$ J. Nitta, T. Akazaki, and H. Takayanagi, Phys. Rev. B 49, 3659 (1994).

${ }^{9}$ S.J.M. Bakker, E. van der Drift, T.M. Klapwijk, H.M. Jaeger, and 
S. Radelaar, Phys. Rev. B 49, 13275 (1994).

${ }^{10}$ P. Xiong, G. Xiao, and R.B. Laibowitz, Phys. Rev. Lett. 71, 1907 (1993).

${ }^{11}$ P.H.C. Magnee, N. van der Post, P.H.M. Kooistra, B.J. van Wees, and T.M. Klapwijk, Phys. Rev. B 50, 4594 (1994).

${ }^{12}$ J. Kutchinsky, R. Taboryski, T. Clausen, C.B. Sorensen, A. Kristensen, P.E. Lindelof, J. Bindslev Hansen, C. Schelde Jacobsen, and J.L. Skov, Phys. Rev. Lett. 78, 931 (1997).

${ }^{13}$ W. Poirier, D. Mailly, and M. Sanquer, Phys. Rev. Lett. 79, 2105 (1997).

${ }^{14}$ G.E. Blonder, M. Tinkham, and T.M. Klapwijk, Phys. Rev. B 25, 4515 (1985).

${ }^{15}$ A.V. Zaitsev, Zh. Eksp. Teor. Fiz. 86, 1724 (1984) [Sov. Phys. JETP 59, 1163 (1984)].

${ }^{16}$ C.W.J. Beenakker, Rev. Mod. Phys. 69, 731 (1997).

${ }^{17}$ C.J. Lambert, J. Phys.: Condens. Matter 3, 6579 (1991).

${ }^{18}$ Y. Takane and H. Ebisawa, J. Phys. Soc. Jpn. 61, 2858 (1992).

${ }^{19}$ C.W.J. Beenakker, Phys. Rev. B 46, 12841 (1992).

${ }^{20}$ C.W.J. Beenakker, B. Rejaei, and J.A. Melsen, Phys. Rev. Lett. 72, 2470 (1994).

${ }^{21}$ G.B. Lesovik, A.L. Fauchere, and G. Blatter, Phys. Rev. B 55, 3146 (1997).

${ }^{22}$ A.I. Larkin and Yu.V. Ovchinnikov, Zh. Eksp. Teor. Fiz. 68, 1915 (1975) [Sov. Phys. JETP 41, 960 (1975)].

${ }^{23}$ A.F. Volkov, A.V. Zaitsev, and T.M. Klapwijk, Physica C 210, 21 (1993)

${ }^{24}$ M.Yu. Kuprianov and V.F. Lukichev, Zh. Eksp. Teor. Fiz. 94, 139 (1988) [Sov. Phys. JETP 67, 1163 (1988)].

${ }^{25}$ K.D. Usadel, Phys. Rev. Lett. 25, 507 (1970).

${ }^{26}$ Yu.V. Nazarov, Phys. Rev. Lett. 73, 1420 (1994).

${ }^{27}$ S. Yip, Phys. Rev. B 52, 3087 (1995).

${ }^{28}$ Yu.V. Nazarov and T.H. Stoof, Phys. Rev. Lett. 76, 823 (1996); T.H. Stoof and Yu.V. Nazarov, Phys. Rev. B 53, 14496 (1996).

${ }^{29}$ A.F. Volkov, N. Allsopp, and C.J. Lambert, J. Phys.: Condens. Matter 8, L45 (1996); A.F. Volkov and H. Takayanagi, Phys. Rev. B 56, 11184 (1997).

${ }^{30}$ A.A. Golubov, F.K. Wilhelm, and A.D. Zaikin, Phys. Rev. B 55, 1123 (1997).

${ }^{31}$ A.F. Volkov and H. Takayanagi, Phys. Rev. B 56, 11184 (1997).

${ }^{32}$ R. Seviour and A.F. Volkov, Phys. Rev. B 61, R9273 (2000).

${ }^{33}$ W. Belzig, F.K. Wilhelm, C. Bruder, G. Schön, and A.D. Zaikin, Superlattices Microstruct. 25, 1251 (1999).

${ }^{34}$ A.A. Golubov and M.Yu. Kuprianov, J. Low Temp. Phys. 70, 83 (1988); W. Belzig, C. Bruder, and G. Schön, Phys. Rev. B 54, 9443 (1996); J.A. Melsen, P.W. Brouwer, K.M. Frahm, and C.W.J. Beenakker, Europhys. Lett. 35, 7 (1996); F. Zhou, P. Charlat, B. Spivak, and B. Pannetier, J. Low Temp. Phys. 110, 841 (1998).

${ }^{35}$ C.J. Lambert, R. Raimondi, V. Sweeney, and A.F. Volkov, Phys. Rev. B 55, 6015 (1997).

${ }^{36}$ E.V. Bezuglyi, E.N. Bratus', V.S. Shumeiko, G. Wendin, and H. Takayanagi, Phys. Rev. B 62, 14439 (2000).

${ }^{37}$ Yu.V. Nazarov, Superlattices Microstruct. 25, 1221 (1999).

${ }^{38}$ Y. Tanaka, A.A. Golubov, and S. Kashiwaya, Phys. Rev. B 68, 054513 (2003).

${ }^{39}$ L.J. Buchholtz and G. Zwicknagl, Phys. Rev. B 23, 5788 (1981); C. Bruder, ibid. 41, 4017 (1990); C.R. Hu, Phys. Rev. Lett. 72, 1526 (1994).

${ }^{40}$ Y. Tanaka and S. Kashiwaya, Phys. Rev. Lett. 74, 3451 (1995); S.
Kashiwaya, Y. Tanaka, M. Koyanagi, and K. Kajimura, Phys. Rev. B 53, 2667 (1996).

${ }^{41}$ Y. Tanaka and S. Kashiwaya, Phys. Rev. B 53, 9371 (1996).

${ }^{42}$ S. Kashiwaya and Y. Tanaka, Rep. Prog. Phys. 63, 1641 (2000), and references therein.

${ }^{43}$ J. Geerk, X.X. Xi, and G. Linker, Z. Phys. B: Condens. Matter 73, 329 (1988).

${ }^{44}$ S. Kashiwaya, Y. Tanaka, M. Koyanagi, H. Takashima, and K. Kajimura, Phys. Rev. B 51, 1350 (1995).

${ }^{45}$ L. Alff, H. Takashima, S. Kashiwaya, N. Terada, H. Ihara, Y. Tanaka, M. Koyanagi, and K. Kajimura, Phys. Rev. B 55, 14757 (1997).

${ }^{46}$ M. Covington, M. Aprili, E. Paraoanu, L.H. Greene, F. Xu, J. Zhu, and C.A. Mirkin, Phys. Rev. Lett. 79, 277 (1997).

${ }^{47}$ J.Y.T. Wei, N.-C. Yeh, D.F. Garrigus, and M. Strasik, Phys. Rev. Lett. 81, 2542 (1998).

${ }^{48}$ I. Iguchi, W. Wang, M. Yamazaki, Y. Tanaka, and S. Kashiwaya, Phys. Rev. B 62, R6131 (2000).

${ }^{49}$ F. Laube, G. Goll, H.v. Löhneysen, M. Fogelström, and F. Lichtenberg, Phys. Rev. Lett. 84, 1595 (2000).

${ }^{50}$ Z.Q. Mao, K.D. Nelson, R. Jin, Y. Liu, and Y. Maeno, Phys. Rev. Lett. 87, 037003 (2001).

${ }^{51}$ Ch. Wälti, H.R. Ott, Z. Fisk, and J.L. Smith, Phys. Rev. Lett. 84, 5616 (2000).

${ }^{52}$ H. Aubin, L.H. Greene, S. Jian, and D.G. Hinks, Phys. Rev. Lett. 89, 177001 (2002).

${ }^{53}$ Z.Q. Mao, M.M. Rosario, K.D. Nelson, K. Wu, I.G. Deac, P. Schiffer, Y. Liu, T. He, K.A. Regan, and R.J. Cava, Phys. Rev. B 67, 094502 (2003).

${ }^{54}$ A. Sharoni, O. Millo, A. Kohen, Y. Dagan, R. Beck, G. Deutscher, and G. Koren, Phys. Rev. B 65, 134526 (2002).

${ }^{55}$ A. Kohen, G. Leibovitch, and G. Deutscher, Phys. Rev. Lett. 90, 207005 (2003).

${ }^{56}$ M.M. Qazilbash, A. Biswas, Y. Dagan, R.A. Ott, and R.L. Greene, Phys. Rev. B 68, 024502 (2003).

${ }^{57}$ J.W. Ekin, Y. Xu, S. Mao, T. Venkatesan, D.W. Face, M. Eddy, and S.A. Wolf, Phys. Rev. B 56, 13746 (1997).

${ }^{58}$ Y. Tanuma, Y. Tanaka, M. Yamashiro, and S. Kashiwaya, Phys. Rev. B 57, 7997 (1998).

${ }^{59}$ Y. Tanuma, Y. Tanaka, M. Ogata, and S. Kashiwaya, J. Phys. Soc. Jpn. 67, 1118 (1998).

${ }^{60}$ Y. Tanuma, Y. Tanaka, M. Ogata, and S. Kashiwaya, Phys. Rev. B 60, 9817 (1999).

${ }^{61}$ Y. Tanuma, Y. Tanaka, and S. Kashiwaya, Phys. Rev. B 64, 214519 (2001).

${ }^{62}$ M. Fogelström, D. Rainer, and J.A. Sauls, Phys. Rev. Lett. 79, 281 (1997); D. Rainer, H. Burkhardt, M. Fogelström, and J.A. Sauls, J. Phys. Chem. Solids 59, 2040 (1998).

${ }^{63}$ M. Matsumoto and H. Shiba, J. Phys. Soc. Jpn. 64, 1703 (1995); M. Matsumoto and H. Shiba, ibid. 64, 4867 (1995).

${ }^{64}$ L.J. Buchholtz, M. Palumbo, D. Rainer, and J.A. Sauls, J. Low Temp. Phys. 101, 1097 (1995).

${ }^{65}$ Y. Tanaka and S. Kashiwaya, Phys. Rev. B 58, 2948 (1998).

${ }^{66}$ M. Yamashiro, Y. Tanaka, and S. Kashiwaya, Phys. Rev. B 56, 7847 (1997).

${ }^{67}$ M. Yamashiro, Y. Tanaka, Y. Tanuma, and S. Kashiwaya, J. Phys. Soc. Jpn. 67, 3224 (1998).

${ }^{68}$ M. Yamashiro, Y. Tanaka, and S. Kashiwaya, J. Phys. Soc. Jpn. 67, 3364 (1998). 
${ }^{69}$ M. Yamashiro, Y. Tanaka, Y. Tanuma, and S. Kashiwaya, J. Phys. Soc. Jpn. 68, 2019 (1999).

${ }^{70}$ C. Honerkamp and M. Sigrist, Prog. Theor. Phys. 100, 53 (1998).

${ }^{71}$ Y. Asano, Y. Tanaka, Y. Matsuda, and S. Kashiwaya, Phys. Rev. B 68, 184506 (2003).

${ }^{72}$ Y. Tanuma, K. Kuroki, Y. Tanaka, and S. Kashiwaya, Phys. Rev. B 64, 214510 (2001).

${ }^{73}$ K. Sengupta, I. Žutić, H.-J. Kwon, V.M. Yakovenko, and S. Das Sarma, Phys. Rev. B 63, 144531 (2001).

${ }^{74}$ Y. Tanuma, K. Kuroki, Y. Tanaka, R. Arita, S. Kashiwaya, and H. Aoki, Phys. Rev. B 66, 094507 (2002).

${ }^{75}$ Y. Tanuma, Y. Tanaka, K. Kuroki, and S. Kashiwaya, Phys. Rev. B 66, 174502 (2002).

${ }^{76}$ Y. Tanaka, H. Tsuchiura, Y. Tanuma, and S. Kashiwaya, J. Phys. Soc. Jpn. 71, 271 (2002).

${ }^{77}$ Y. Tanaka, Y. Tanuma, K. Kuroki, and S. Kashiwaya, J. Phys. Soc. Jpn. 71, 2102 (2002).

${ }^{78}$ Y. Tanaka, H. Itoh, H. Tsuchiura, Y. Tanuma, J. Inoue, and S. Kashiwaya, J. Phys. Soc. Jpn. 71, 2005 (2002).

${ }^{79}$ Yu.S. Barash, M.S. Kalenkov, and J. Kurkijarvi, Phys. Rev. B 62, 6665 (2000).

${ }^{80}$ J.-X. Zhu, B. Friedman, and C.S. Ting, Phys. Rev. B 59, 9558 (1999).

${ }^{81}$ S. Kashiwaya, Y. Tanaka, N. Yoshida, and M.R. Beasley, Phys. Rev. B 60, 3572 (1999).

${ }^{82}$ I. Zutic and O.T. Valls, Phys. Rev. B 60, 6320 (1999).

${ }^{83}$ N. Yoshida, Y. Tanaka, J. Inoue, and S. Kashiwaya, J. Phys. Soc. Jpn. 68, 1071 (1999).

${ }^{84}$ T. Hirai, N. Yoshida, Y. Tanaka, J. Inoue, and S. Kashiwaya, J. Phys. Soc. Jpn. 70, 1885 (2001).

${ }^{85}$ N. Yoshida, H. Itoh, T. Hirai, Y. Tanaka, J. Inoue, and S. Kashiwaya, Physica C 367, 135 (2002).

${ }^{86}$ T. Hirai, Y. Tanaka, N. Yoshida, Y. Asano, J. Inoue, and S. Kashiwaya, Phys. Rev. B 67, 174501 (2003).

${ }^{87}$ Y. Tanaka and S. Kashiwaya, J. Phys. Soc. Jpn. 68, 3485 (1999).

${ }^{88}$ Y. Tanaka and S. Kashiwaya, J. Phys. Soc. Jpn. 69, 1152 (2000).

${ }^{89}$ Y. Tanaka and S. Kashiwaya, Phys. Rev. B 53, 11957 (1996); 56, 892 (1997).

${ }^{90}$ E. Il'ichev, V. Zakosarenko, R.P.J. Ijsselsteijn, V. Schultze, H.-G. Meyer, H.E. Hoenig, H. Hilgenkamp, and J. Mannhart, Phys. Rev. Lett. 81, 894 (1998).

${ }^{91}$ Y. Asano, Phys. Rev. B 63, 052512 (2001).

${ }^{92}$ Y. Asano, Phys. Rev. B 64, 014511 (2001).

${ }^{93}$ Y. Asano, Phys. Rev. B 64, 224515 (2001).

${ }^{94}$ Y. Asano, J. Phys. Soc. Jpn. 71, 905 (2002).

${ }^{95}$ Y. Asano, Y. Tanaka, M. Sigrist, and S. Kashiwaya, Phys. Rev. B 67, 184505 (2003).

${ }^{96}$ Y.S. Barash, H. Burkhardt, and D. Rainer, Phys. Rev. Lett. 77, 4070 (1996).
${ }^{97}$ E. Il'ichev, M. Grajcar, R. Hlubina, R.P.J. IJsselsteijn, H.E. Hoenig, H.-G. Meyer, A. Golubov, M.H.S. Amin, A.M. Zagoskin, A.N. Omelyanchouk, and M.Yu. Kuprianov, Phys. Rev. Lett. 86, 5369 (2001).

${ }^{98}$ G. Testa, A. Monaco, E. Esposito, E. Sarnelli, D.-J. Kang, E.J. Tarte, S.H. Mennema, and M.G. Blamire, cond-mat/0310727 (unpublished).

${ }^{99}$ Y. Tanaka, T. Hirai, K. Kusakabe, and S. Kashiwaya, Phys. Rev. B 60, 6308 (1999).

${ }^{100}$ T. Hirai, K. Kusakabe, and Y. Tanaka, Physica C 336, 107 (2000); K. Kusakabe and Y. Tanaka, ibid. 367, 123 (2002); K. Kusakabe and Y. Tanaka, J. Phys. Chem. Solids 63, 1511 (2002).

${ }^{101}$ N. Stefanakis, Phys. Rev. B 64, 224502 (2001).

${ }^{102}$ Z.C. Dong, D.Y. Xing, and J. Dong, Phys. Rev. B 65, 214512 (2002); Z.C. Dong, D.Y. Xing, Z.D. Wang, Z. Zheng, and J. Dong, ibid. 63, 144520 (2001).

${ }^{103}$ M.H.S. Amin, A.N. Omelyanchouk, and A.M. Zagoskin, Phys. Rev. B 63, 212502 (2001).

${ }^{104}$ S.-T. Wu and C.-Y. Mou, Phys. Rev. B 66, 012512 (2002).

${ }^{105}$ A.A. Golubov and M.Y. Kuprianov, Pis'ma Zh. Éksp. Teor. Fiz. 69, 242 (1999) [JETP Lett. 69, 262 (1999)]; 67, 478 (1998) [67, 501 (1998)].

${ }^{106}$ A. Poenicke, Yu.S. Barash, C. Bruder, and V. Istyukov, Phys. Rev. B 59, 7102 (1999); K. Yamada, Y. Nagato, S. Higashitani, and K. Nagai, J. Phys. Soc. Jpn. 65, 1540 (1996).

${ }^{107}$ T. Lück, U. Eckern, and A. Shelankov, Phys. Rev. B 63, 064510 (2002).

${ }^{108}$ Y. Tanaka, Y.V. Nazarov, and S. Kashiwaya, Phys. Rev. Lett. 90, 167003 (2003).

${ }^{109}$ Y. Tanaka and S. Kashiwaya, cond-mat/0308123 (unpublished).

${ }^{110}$ H. Kashiwaya, A. Sawa, S. Kashiwaya, H. Yamasaki, M. Koyanagi, I. Kurosawa, Y. Tanaka, and I. Iguchi, Physica C 357-360, 1610 (2001); H. Kashiwaya, I. Kurosawa, S. Kashiwaya, A. Sawa, and Y. Tanaka, Phys. Rev. B 68, 054527 (2003).

${ }^{111}$ T.M. Klapwijk, G.E. Blonder, and M. Tinkham, Physica B \& C 109-110, 1657 (1982).

${ }^{112}$ M. Octavio, M. Tinkham, G.E. Blonder, and T.M. Klapwijk, Phys. Rev. B 27, 6739 (1983).

${ }^{113}$ G.B. Arnlod, J. Low Temp. Phys. 68, 1 (1987); U. Gunsenheimer and A.D. Zaikin, Phys. Rev. B 50, 6317 (1994).

${ }^{114}$ E.N. Bratus', V.S. Shumeiko, and G. Wendin, Phys. Rev. Lett. 74, 2110 (1995); D. Averin and A. Bardas, ibid. 75, 1831 (1995); J.C. Cuevas, A. Martin-Rodero, and A.L. Yeyati, Phys. Rev. B 54, 7366 (1996).

${ }^{115}$ A. Bardas and D.V. Averin, Phys. Rev. B 56, 8518 (1997); A.V. Zaitsev and D.V. Averin, Phys. Rev. Lett. 80, 3602 (1998).

${ }^{116}$ A.V. Zaitsev, Physica C 185-189, 2539 (1991).

${ }^{117}$ E.V. Bezuglyi, E.N. Bratus', V.S. Shumeiko, and G. Wendin, Phys. Rev. Lett. 83, 2050 (1990). 